OPEN ACCESS

Edited by:

Rashika El Ridi,

Cairo University, Egypt

Reviewed by:

Mohamed Labib Salem,

Tanta University, Egypt

Afzal A. Siddiqui,

Texas Tech University Health

Sciences Center, USA

Lúcia Helena Faccioli,

Universidade de São Paulo, Brazil

${ }^{*}$ Correspondence:

Claudia Carvalho-Queiroz,

Stockholm University,

Department of Molecular

Biosciences, The Wenner-Gren Institute, SE-106, 91 Stockholm,

Sweden

claudia.queiroz@su.se

Philip T. LoVerde,

Departments of Biochemistry and

Pathology, University of Texas Health

Sciences Center, 7703 Floyd Curl

Drive, San Antonio,

TX 78229-3800, USA

loverde@uthscsa.edu

${ }^{\dagger}$ Present address:

Claudia Carvalho-Queiroz,

Department of Molecular

Biosciences, Wenner-Gren Institute

(MBW), Stockholm University,

Stockholm, Sweden

Nejat K. Egilmez,

Department of Microbiology and Immunology, School of Medicine,

University of Louisville,

Louisville, KY, USA

Specialty section:

This article was submitted to Immunotherapies and Vaccines,

a section of the journal

Frontiers in Immunology

Received: 23 January 2015

Accepted: 16 May 2015

Published: 02 June 2015

Citation:

Carvalho-Queiroz C, Nyakundi R, Ogongo P, Rikoi H, Egilmez NK, Farah IO, Kariuki TM and LoVerde PT

(2015) Protective potential of antioxidant enzymes as vaccines for

schistosomiasis in a non-human

primate model. Front. Immunol. 6:273.

doi: 10.3389/fimmu.2015.00273

\section{Protective potential of antioxidant enzymes as vaccines for schistosomiasis in a non-human primate model}

\author{
Claudia Carvalho-Queiroz ${ }^{1,2, *}$, Ruth Nyakundi ${ }^{3}$, Paul Ogongo $^{3}$, Hitler Rikoi ${ }^{3}$, \\ Nejat K. Egilmez ${ }^{4 \dagger}$, Idle O. Farah ${ }^{3}$, Thomas M. Kariuki ${ }^{3}$ and Philip T. LoVerde ${ }^{1,2 *}$ \\ ${ }^{1}$ Department of Biochemistry, University of Texas Health Science Center, San Antonio, TX, USA, ${ }^{2}$ Department of Pathology, \\ University of Texas Health Science Center, San Antonio, TX, USA, ${ }^{3}$ Institute of Primate Research, National Museums of \\ Kenya, Nairobi, Kenya, ${ }^{4}$ Department of Microbiology and Immunology, School of Medicine and Biomedical Sciences, \\ University at Buffalo, Buffalo, NY, USA
}

Schistosomiasis remains a major cause of morbidity in the world. The challenge today is not so much in the clinical management of individual patients, but rather in population-based control of transmission in endemic areas. Despite recent largescale efforts, such as integrated control programs aimed at limiting schistosomiasis by improving education and sanitation, molluscicide treatment programs and chemotherapy with praziquantel, there has only been limited success. There is an urgent need for complementary approaches, such as vaccines. We demonstrated previously that anti-oxidant enzymes, such as $\mathrm{Cu}$-Zn superoxide dismutase (SOD) and glutathione S peroxidase (GPX), when administered as DNA-based vaccines induced significant levels of protection in inbred mice, greater than the target $40 \%$ reduction in worm burden compared to controls set as a minimum by the $\mathrm{WHO}$. These results led us to investigate if immunization of non-human primates with antioxidants would stimulate an immune response that could confer protection as a prelude study for human trials. Issues of vaccine toxicity and safety that were difficult to address in mice were also investigated. All baboons in the study were examined clinically throughout the study and no adverse reactions occurred to the immunization. When our outbred baboons were vaccinated with two different formulations of SOD (SmCT-SOD and SmEC-SOD) or one of GPX (SmGPX), they showed a reduction in worm number to varying degrees, when compared with the control group. More pronounced, vaccinated animals showed decreased bloody diarrhea, days of diarrhea, and egg excretion (transmission), as well as reduction of eggs in the liver tissue and in the large intestine (pathology) compared to controls. Specific IgG antibodies were present in sera after immunizations and 10 weeks after challenge infection compared to controls. Peripheral blood mononuclear cells, mesenteric, and inguinal node cells from vaccinated animals proliferated and produced high levels of cytokines and chemokines in response to crude and recombinant antigens compared with controls. All together, these data demonstrate the potential of antioxidants as a vaccine in a non-human primate model.

Keywords: Schistosoma mansoni, antioxidants, vaccine, anti-fecundity, baboon 


\section{Introduction}

Schistosomiasis remains a major cause of morbidity in the world. The disease is endemic in 76 countries of the world where it affects some 200 million people (1). Substantial research has established that school-aged children in endemic areas are at significant risk of serious disease resulting from infection with schistosomes (2, 3). Lack of access to clean water and sanitation and inadequate personal hygiene are important shared risk factors for these infections (4).

Currently, chemotherapy with praziquantel (PZQ) is the preferred treatment for schistosomiasis (5-8). Control programs based on mass chemotherapy are complicated by rapid and frequent re-infection and the difficulties and expense of maintaining these programs over a long term (9-12). There is evidence that schistosomes develop drug resistance against PZQ in some regions $(13,14)$, and there is additional evidence for serious rebound morbidity if regular and periodic treatments are interrupted (15-17). Despite recent large-scale efforts, integrated control programs aimed at limiting schistosomiasis by improving education and sanitation, molluscicide treatment programs to reduce the population of the intermediate snail host, and chemotherapy have had limited success (18). Effective control of schistosomiasis could prevent up to 130,000 deaths and avert up to 25 million disability adjusted life years lost annually (19). The challenge today is not so much in the clinical management of individual patients, but rather in population-based control of transmission (and consequently, of morbidity) in endemic areas. Because current control strategies employing chemotherapy with PZQ have not reduced transmission and morbidity to acceptable levels, there is an urgent need for complementary approaches, such as vaccines for schistosomiasis $(20,21)$.

Our own studies have focused on antioxidant enzymes as vaccine candidates [recently reviewed by Huang et al. (22)]. We hypothesized that antioxidants play a role in protecting the adult worms from damage derived from reactive oxygen species (23-26). To begin to test this hypothesis, we demonstrated that expression of the schistosome antioxidant enzymes [Cu-Zn superoxide dismutase (SOD); glutathione $S$ peroxidase (GPX)] is developmentally regulated such that the lowest levels of gene expression (as measured by transcription) and enzyme specific activity were in the larval stages, the most susceptible to immune killing, and highest in adult worms, the least susceptible to immune elimination $(23,25$, 27-29). To provide direct evidence that antioxidant enzymes were important in immune evasion (25) and thus were viable candidate vaccines, we used DNA vaccination strategies to demonstrate the efficacy of DNA constructs encoding either $\mathrm{Cu} / \mathrm{Zn}$ cytosolic superoxide dismutase (SmCT-SOD), signal peptide-containing SOD (SmSP-SOD, also known as SmEC-SOD), or glutathione peroxidase (SmGPX) to be protective against Schistosoma mansoni infection in a murine challenge model. Employing different doses of plasmid cDNA constructs, mice exhibited a significant level of worm burden reduction when challenged with $S$. mansoni cercariae after immunization with SmCT-SOD (54\%) and SmGPX (43.4\%) from six independent experiments (29).

The WHO has identified several candidate vaccine antigens for independent evaluation, but none fulfilled the required standards in trials with mice. One of the criteria was a worm burden reduction of $>40 \%$ in a murine challenge model (30). We have demonstrated that both SmCT-SOD and SmGPX were each capable as DNA-based (plasmid or vaccinia virus vehicles) vaccines to consistently induce significant levels of protection in an S. mansoni murine-challenge model, greater than the target $40 \%$ reduction in worm burden compared to control set as a minimum by the WHO. We also demonstrated in a mouse model that DNA encoding SmCT-SOD as a vaccine is able to significantly reduce worm burden by targeting adult $S$. mansoni worms 21 days and older (31).

The above results with the antioxidant enzymes in a murine model of S. mansoni encouraged us to investigate if immunization of non-human primates with antioxidants would stimulate an immune response that would be safe and that could confer protection.

\section{Materials and Methods}

\section{Animals and Screening}

Young wild caught Olive Baboons (Papio cynocephalus anubis), both males and females aged 4-5 years, were from schistosomiasis non-endemic areas and habituated and quarantined for 90 days in accordance with good animal welfare standards. The baboons were screened for the presence of several infections and parasites (such as malaria, tuberculosis, leishmaniasis, nematodes, etc.) before they were assigned to the study. For assessing the presence of current Schistosoma infections, the fecal material was examined for the presence of eggs and the serum from each animal was checked for the presence of antibodies against soluble egg antigens (SEA). Positive animals were excluded from our study or when necessary successfully treated using ivermectin and/or metro-nidazole as appropriate, months before the start of immunization. Detailed hematological tests certified all animals were in excellent health during their quarantine period. The animals were housed isolated at the facilities of the Institute of Primate Research (IPR), Karen, Nairobi, Kenya. Ethical clearance for these studies was obtained from the Institute for Primate Research IACUC (IPR/ SRP3/2004) and the University of Texas Health Science Center at San Antonio IACUC (08039x).

\section{Preparation of the Antioxidant Vaccines}

cDNA containing the entire open reading frames of SmCT-SOD, SmEC-SOD, SmGPX were previously cloned into the eukaryotic expression vectors pcDNAI/Amp (pc) $(31,32)$ and VR1055 (Vical, San Diego, CA, USA) (33). Each plasmid preparation was then purified by double gradient centrifugation in $\mathrm{CsCl}_{2}$, dialyzed against PBS to remove the $\mathrm{CsCl}_{2}$, ethanol precipitated, and re-suspended in sterile sucrose (25\% in PBS). The last boost consisted of the respective recombinant proteins incorporated into microspheres made of polylactic acid (PLA). Only recombinant proteins were incorporated into PLA microspheres, as previously described by us (32). Of note, the following terms are used interchangeably throughout the study: SmCT-SOD or CTSOD; SmEC-SOD or ECSOD; SmGPX or GPX; and Vector Only or Control. 


\section{Immunizations and Challenge}

Two vaccination experimental protocols were carried out: Experiment 1 (Figure 1A) was for safety and toxicity evaluation after vaccination, while Experiment 2 (Figure 2A) for assessment of the efficacy of vaccination upon challenge with S. mansoni. In Experiment 1 (Figure 1A), groups of five olive baboons received $500 \mu \mathrm{g}$ of purified DNA via two intramuscular (quadriceps) injections with a 26-gage needle in each leg. Each group received a second and third $500 \mu \mathrm{g}$ dose of their respective DNA, 4 weeks apart. A fourth dose of $500 \mu \mathrm{g}$ of recombinant proteins encapsulated in PLA microspheres as described (32) was given 4 weeks after the third injection. The control animals were primed and boosted with empty vector DNA, while the last boost consisted of empty PLA microspheres in PBS instead of recombinant proteins.

In Experiment 2 (Figure 2A), groups of five 4- to 5-year-old olive baboons were primed with $1000 \mu \mathrm{g}$ of purified DNA, boosted once with $1000 \mu \mathrm{g}$ doses of their respective purified DNA, and once with $1000 \mu \mathrm{g}$ of the respective recombinant protein encapsulated in PLA microspheres, with all immunizations 4 weeks apart from each other. The control animals were primed and boosted with empty vector DNA, while the last boost consisted of empty PLA microspheres in PBS instead of recombinant proteins. The baboons were then anesthetized using a mixture of xylazine ( $2 \%$ Rompun $^{\mathrm{mm}}$ ) and Ketamine hydrochloride (100 mg/ml; Agrar Holland BV, Soest, The Netherlands) at a dose of $10 \mathrm{mg} / \mathrm{kg}$ body weight. After shaving the groin areas of the baboons, 600 cercariae (Kenyan strain) in suspension from individual beakers were poured into a groin pouch and left to penetrate via the skin for $30 \mathrm{~min}$.

\section{Blood Collection for Hematology, Cell, and Serum Isolation}

All animals were anesthetized with ketamine hydrochloride (10 mg/kg body weight) prior to blood collection. Then, a $10 \mathrm{ml}$ syringe with a 21-gauge needle was used to draw blood from the femoral vein, where it was placed into an EDTA treated tube for hematology (Experiment 1) or used for serum collection (Experiment 2). In addition, $50 \mathrm{ml}$ syringes containing $20 \mathrm{ml}$ of Alsever's anti-coagulant solution (Sigma) and fitted with a 21-gauge needle were used to draw $20 \mathrm{ml}$ of blood to a total volume of $40 \mathrm{ml}$ blood mixture from the opposite limb (Experiment 2).

In the Experiment 1, as depicted in Figure 1A, the blood was drawn every 2 weeks (with exception of the last time point in which blood was taken 3 weeks after last boost). The eight time points were: week 0 (pre-immune, PI), week 2 (2 weeks post-priming), week 4 (pre-1st boost), week 6 ( 2 weeks post-1st boost), week 8 (pre-2nd boost), week 10 (2 weeks post-2nd boost), week 12 (pre-3rd boost), and week 15 (3 weeks post-3rd Boost). In the Experiment 2 (Figure 2A), the blood was drawn at three timepoints: week 0 (pre-immune, PI), week 12 (pre-challenge, PC), and week 22 (pre-perfusion, PP).

\section{Clinical Assessment for Safety and Toxicity}

The baboons were monitored daily for appetite, behavior, and demeanor, and tested at 2-week intervals for temperature, weight measurements, complete blood counts, and leukocyte differentials at baseline (week 0) and after all immunizations.

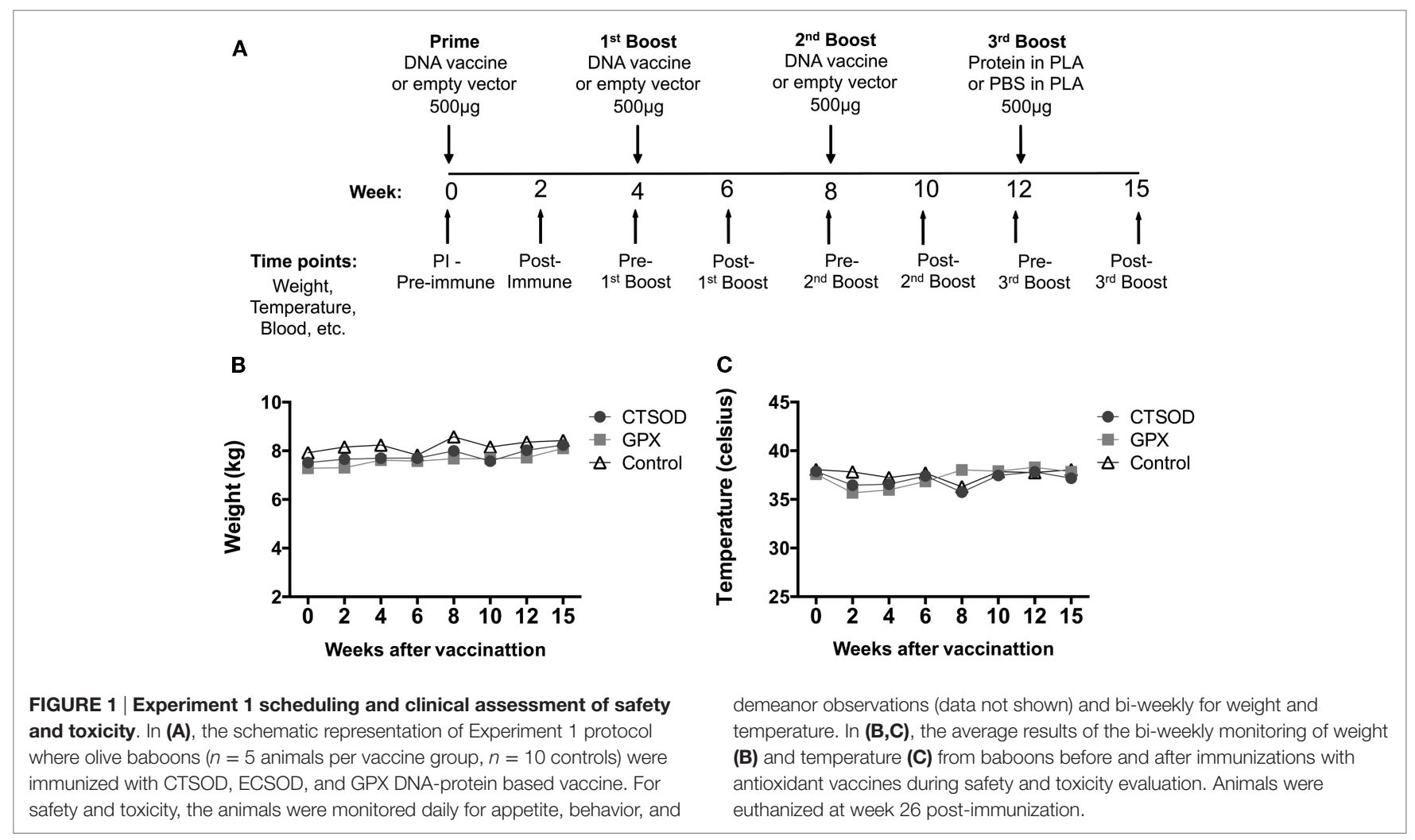



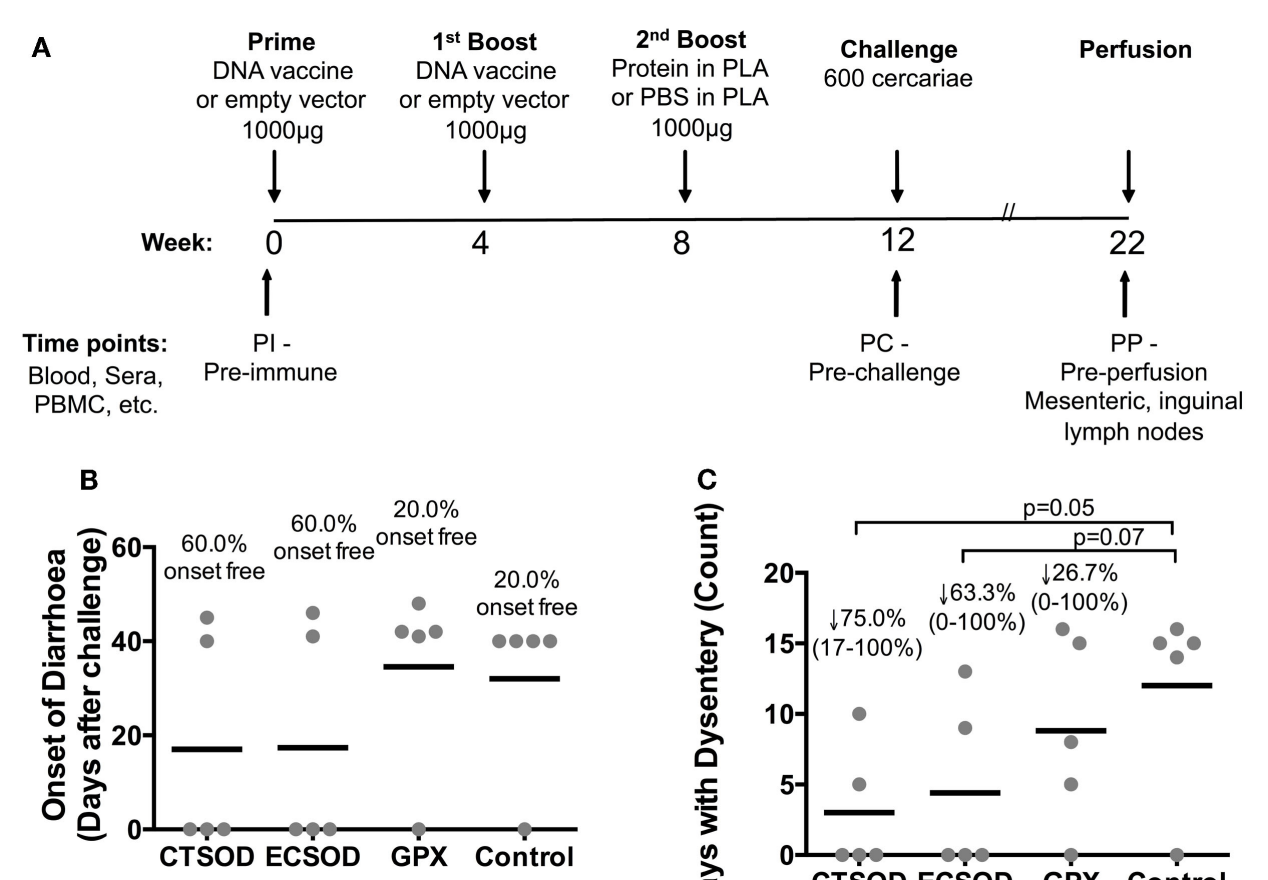

FIGURE 2 | Experiment 2 scheduling and clinical assessment after challenge infection. In (A), the schematic representation of the Experiment 2 protocol where olive baboons were immunized with CTSOD, ECSOD, and GPX DNA-protein based vaccine ( $n=5$ animals per each group), and further challenged with $600 \mathrm{~S}$. mansoni cercariae. Animals were also assessed for safety and toxicity of vaccination (data not shown). In (B,C), commence of diarrhea and duration of dysentery in days after challenge infection. Individual

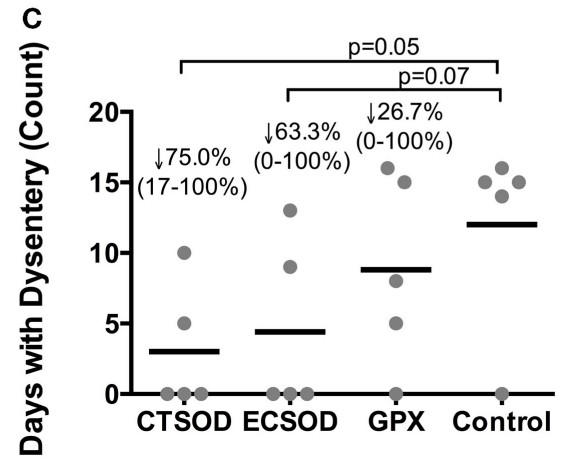

values (sphere); mean average (dash). The percentage of animals that did not suffer diarrhea is represented in (B). In (C), the percentage of reduction (represented by the arrow), the range (minimal-maximal, represented between brackets), and the statistical $p$-value were expressed in comparison to the mean average of the Control group. Non-parametric (Kruskal-Wallis, MannWhitney) tests were applied. Values were considered statistically significant when $p<0.05$

\section{Perfusion}

Approximately 10 weeks post-challenge, the baboons were euthanized by intravenous administration of heparinized sodium pentobarbital. For each animal, the viscera were exposed after a median incision. The gross pathology of the liver, intestines, and mesenteric lymph nodes was evaluated. Perfusion of worms from the mesenteric vasculature and the liver was achieved after the administration of citrated saline through the abdominal aorta, as described previously (34), and adult worms were counted. To ensure that all worms were recovered, during the perfusion, the mesenteric veins were manually searched for schistosome worms. The worms were counted, and as an indicator of efficacy, the mean percent protection observed in the experimental groups was determined as the decrease in worm burden of each animal compared to the mean recovery of the control groups (35).

\section{Parasitological Parameters}

The weekly assessment of eggs per gram of feces (EPG) from vaccinated and control baboons commencing 6 weeks after S. mansoni cercariae challenge was determined by the thick smear Kato-Katz microscope technique (36). For extraction and determination of eggs present in organs, $10 \%$ tissue samples (by weight) of liver and intestines were digested overnight in $5 \% \mathrm{KOH}$, as described previously (37). The eggs were counted and the percentage of reduction

in egg excretion in stool or tissues calculated in relation to the mean average of the control group.

\section{Enzyme-Linked Immunosorbent Assay}

The titers of IgG antibodies in the baboon sera against recombinant antigens derived from S. mansoni were determined by ELISA as described with minor modifications (32). Flat-bottom 96-well polystyrene plates (Maxisorp Nunc, GIBCO, Scotland) were coated overnight at $4^{\circ} \mathrm{C}$ with $50 \mu \mathrm{l}$ of antigens diluted in PBS $0.15 \mathrm{M}, \mathrm{pH} 7.2$ (PBS) at concentration of $5 \mu \mathrm{g} / \mathrm{ml}$. After washing (automatic ELISA Washer - MR 5000, Dynatech) and blocking, $50 \mu \mathrm{l} /$ well of doubling dilution of individual serum or pooled sera diluted in PBS-Tween 20, 0.05\% PBS-T were added to each plate and the plates incubated overnight at $4^{\circ} \mathrm{C}$. After washing, $50 \mu \mathrm{l} /$ well of unconjugated goat anti-monkey IgG antibodies (Abd Serotec) were added and the plates were incubated $1 \mathrm{~h}$ at $37^{\circ} \mathrm{C}$. After the incubation, the plates were washed, and $50 \mu \mathrm{l}$ of alkaline-phosphatase conjugated donkey anti-goat IgG antibodies (Abd Serotec) were added to those plates with unconjugated antibody, and the plates were incubated for another $1 \mathrm{~h}$ at $37^{\circ} \mathrm{C}$. Detection of reactivity was performed by using $100 \mu \mathrm{l} /$ well of pNPP in diethanolamine (DEA) buffer ( $p$ NPP Microwell Substrate System, KPL) and the absorbance measured at intervals at $405 \mathrm{~nm}$ by an automatic ELISA Reader (BIORAD). All the 
assay conditions were previously set up for optimal concentrations through checkerboard and titration curves and all the reagents for antibody detection used in this assay were shown to be clear of non-specific reactions.

\section{Isolation of Peripheral Blood Mononuclear Cells and Tissue Cells for Recall Proliferation and Cytokine Culture}

The mixture of blood/Alsever's fluid was layered in two tubes containing $10 \mathrm{ml}$ of density gradient solution (Ficoll-Paque ${ }^{\mathrm{Tx}}$ Research Grade, Pharmacia, Uppsala, Sweden) and centrifuged at $2000 \mathrm{rpm}$ for $20 \mathrm{~min}$ at room temperature. The peripheral blood mononuclear cells (PBMCs) were recovered from the gradient interphase of plasma and red blood cells, washed twice in RPMI 1640 medium containing $80 \mu \mathrm{g} / \mathrm{ml}$ gentamycin (Life Technologies, UK) by centrifuging $2000 \mathrm{rpm}$ for $10 \mathrm{~min}$ for the first time, and $1500 \mathrm{rpm}$ for $10 \mathrm{~min}$ for the second time. The pellets were re-suspended in $10 \mathrm{ml}$ of RPMI 1640 containing $10 \mu \mathrm{g} / \mathrm{ml}$ of Polymixin B, 10\% FCS (GIBCO, Paisley, UK), $80 \mu \mathrm{g} / \mathrm{ml}$ gentamycin solution, $1 \%$ glutamine $(200 \mathrm{mM})$, and $25 \mathrm{mM}$ HEPES. For cells derived from spleen, mesenteric, and inguinal lymph nodes, the tissues were disrupted after having been pressed over a $40 \mu \mathrm{m}$ cell strainer with a help of a syringe plunge. The cells were washed once in RPMI 1640 medium containing $80 \mu \mathrm{g} / \mathrm{ml}$ gentamycin (Life Technologies, UK) by centrifuging $2000 \mathrm{rpm}$ for $10 \mathrm{~min}$ before the red blood cells were lysed for $10 \mathrm{~min}$ with lysing solution. After two more washes, the pellet was also re-suspended in $10 \mathrm{ml}$ of RPMI 1640 containing $10 \mu \mathrm{g} / \mathrm{ml}$ of Polymixin B, 10\% FCS (GIBCO, Paisley, UK), $80 \mu \mathrm{g} /$ $\mathrm{ml}$ gentamycin solution, $1 \%$ glutamine $(200 \mathrm{mM})$, and $25 \mathrm{mM}$ HEPES. Viable cells were counted in a hemocytometer chamber using Trypan Blue dye exclusion (Sigma, St Louis, MO, USA). After cell count adjustment, $2 \times 10^{5}$ cells were dispensed with $200 \mu \mathrm{l}$ of RPMI 1640 containing 10\% FCS (GIBCO, Paisley, UK), $80 \mu \mathrm{g} / \mathrm{ml}$ gentamycin solution, $1 \%$ glutamine $(200 \mathrm{mM})$, and $25 \mathrm{mM}$ Hepes into triplicate wells of a 96-well microtiter plates containing $20 \mu \mathrm{l}$ of parasite antigen at $5 \mu \mathrm{g} / \mathrm{ml}$ (SWAP, SEA), $10 \mu \mathrm{g} / \mathrm{ml}$ (recombinant antigens), or $5 \mu \mathrm{g} / \mathrm{ml}$ of Con A. Plates were cultured at $37^{\circ} \mathrm{C}, 5 \%$ $\mathrm{CO}_{2}$ for $72 \mathrm{~h}$, when $20 \mu \mathrm{l}$ of $0.5 \mu \mathrm{Ci}$ of ${ }^{3} \mathrm{H}$ thymidine was added per well. After incubation for another 18-20 h, the cells were harvested onto fiberglass filter papers before the thymidine incorporation was measured using $2 \mathrm{ml}$ of scintillation fluid per filter in a Beta counter. Results were expressed as stimulation index (S.I.), the ratio of the mean counts per minute $(\mathrm{cpm})$ of triplicate culture cells taken up in the presence of the antigen over those obtained with medium alone.

For cytokine cultures, $2 \times 10^{6}$ cells were dispensed with $1000 \mu \mathrm{l}$ of RPMI 1640 containing 10\% FCS (GIBCO, Paisley, UK), $80 \mu \mathrm{g} /$ $\mathrm{ml}$ gentamycin solution, $1 \%$ glutamine $(200 \mathrm{mM})$, and $25 \mathrm{mM}$ HEPES into each well of 48-well tissue culture plates containing $20 \mu \mathrm{l}$ of parasite antigen at $25 \mu \mathrm{g} / \mathrm{ml}$ (Schistosome Soluble Worm Antigen Preparation, SWAP; Soluble Egg Antigen, SEA), $50 \mu \mathrm{g} /$ $\mathrm{ml}$ (recombinant antigens), or $25 \mu \mathrm{g} / \mathrm{ml}$ of Con A. After $72 \mathrm{~h}$ of incubation at $37^{\circ} \mathrm{C}, 5 \% \mathrm{CO}_{2}$, about $800 \mu \mathrm{l}$ of supernatant/well were harvested and stored at $-70^{\circ} \mathrm{C}$ until use. Freezing medium as $80 \% \mathrm{FCS} / 20 \%$ dimethyl sulfoxide (DMSO) was used in a drop wise manner in order to keep the remaining cells in cryo-freezing vials at $1 \times 10^{7} / \mathrm{ml}$ concentration inside a liquid nitrogen tank.

\section{Analysis of Soluble Cytokines and Chemokines Secretion After Antigenic Stimulation}

Harvested supernatants derived from PBMC cultures after stimulation with crude and recombinant antigens were assayed simultaneously using a 23-plex Non-Human Primate Cytokine/ Chemokine Immunoassay Milliplex Kit (Millipore, Billerica, MA, USA) according to the manufacturer's instructions, including quality controls. Briefly, $50 \mu \mathrm{l}$ of standards or samples were incubated with multi-cytokine beads for $2 \mathrm{~h}$ in the dark, and following washes with a vacuum manifold (Millipore, Billerica, MA, USA) with biotinylated reporter for $1.5 \mathrm{~h}$. The plates were then incubated with Streptavidin-Phycoerythrin for $30 \mathrm{~min}$ before the reaction was stopped for data collection in the Luminex 200 instrument using Luminex IS 2.3 software (Luminex Corporation, Austin, TX, USA) with a minimum of 50 beads per analyte. The resulting mean fluorescence intensity (MFI) was normalized and analyzed through the BeadView Multiplex Data Analysis Software version 1.0 (Millipore, Billerica, MA, USA), and expressed in $\mathrm{pg} / \mathrm{ml}$. The following cytokines and chemokines were assayed: IFN- $\gamma$, IL-12, IL-4, IL-6, IL-17A, CCL3 (MIP-1 $\alpha$ ), IL-5, IL-13, CCL2 (MCP-1), IL-1 $\beta$, IL-2, IL-15, CCL4 (MIP-1 $\beta)$, TNF- $\alpha$, IL-10, TGF- $\alpha$, IL-1Ra, IL-8, GM-CSF, sCD40L, VEGF, G-CSF, IL-18.

\section{Statistical Analysis}

Statistical analyses were performed using GraphPad Prism (Prism 6 for Mac OS X) software. Parametric tests (ANOVA, student's $t$-tests) were used after log-transformation with corrections for multiple analyses. Otherwise, non-parametric (Kruskal-Wallis, Mann-Whitney) tests were applied. Values were considered statistically significant when $p<0.05$, and assigned ${ }^{\star} p=0.01-0.05$; ${ }^{* *} p=0.001-0.01$; and ${ }^{* *} p=<0.001$.

\section{Results}

\section{Safety and Toxicity (Experiment 1)}

As noted in Section "Immunizations and Challenge" and in Figure 1A, the Experiment 1 was for safety and toxicity evaluation after vaccination. All animals in the study were examined clinically throughout the study. The animals appeared healthy, eating normally without any physical signs of toxicity. The weight (Figure 1B) and temperature (Figure 1C) of each animal were normal throughout the study. In addition, as shown in Tables 1 and 2 , the hematological values of the vaccinated animals remained within the normal limits throughout the observation period and there were no other clinical adverse signs. In addition, we examined the vaccine injection sites following immunization and did not observe any significant irritation at the vaccination site i.e., baboons did not experience any muscle transient induration, granulomas, abscesses, ulcers, cutaneous erythema, inguinal lymphadenopathy,or skin swelling at the vaccination sites (data not shown). In a similar manner, we examined baboons for adverse events due to vaccination in Experiment 2. There were none.

\section{Diarrhea, Duration of Dysentery, and Egg Excretion (Experiment 2)}

The safety of our vaccine candidates encouraged us to proceed with Experiment 2 (outlined in Figure 2A), where groups of five 
TABLE 1 | Summary of effects of vaccination in baboons (Experiment 1) ${ }^{\text {a }}$.

\begin{tabular}{|c|c|c|c|c|c|c|c|c|}
\hline Week & & Time point & WBC $\left(\times 10^{3}\right)$ & Neu (\%) & Eos (\%) & Baso (\%) & Lymp (\%) & Mono (\%) \\
\hline \multirow{8}{*}{ 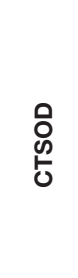 } & 0 & $\mathrm{PI}$ & $8.84 \pm 2.53$ & $61.2 \pm 3.7$ & $1 \pm 0.71$ & $0 \pm 0$ & $34.2 \pm 0.96$ & $2.6 \pm 1.14$ \\
\hline & 2 & Post-I & $14.98 \pm 5.09$ & $59.2 \pm 6.94$ & $1.8 \pm 1.30$ & $0 \pm 0$ & $35 \pm 9.08$ & $3.8 \pm 2.17$ \\
\hline & 4 & Pre-boost 1 & $12.66 \pm 2.0$ & $58.8 \pm 12.58$ & $1.2 \pm 1.30$ & $0 \pm 0$ & $38.8 \pm 11.99$ & $1.2 \pm 0.45$ \\
\hline & 6 & Post-boost 1 & $11.48 \pm 2.74$ & $45.6 \pm 10.78$ & $1.2 \pm 2.17$ & $0 \pm 0$ & $49.8 \pm 10.92$ & $2.6 \pm 1.34$ \\
\hline & 8 & Pre-boost 2 & $10.68 \pm 2.95$ & $38.2 \pm 15.75$ & $2.2 \pm 2.17$ & $0 \pm 0$ & $59 \pm 16.73$ & $0.6 \pm 0.89$ \\
\hline & 10 & Post-boost 2 & $16.86 \pm 4.77$ & $41.6 \pm 9.18$ & $3.2 \pm 1.64$ & $0 \pm 0$ & $55 \pm 8.34$ & $0.2 \pm 0.45$ \\
\hline & 12 & Pre-boost 3 & $12.52 \pm 4.04$ & $50.4 \pm 17.56$ & $2.4 \pm 1.67$ & $0 \pm 0$ & $46.4 \pm 16.52$ & $0.6 \pm 0.89$ \\
\hline & 15 & Post-boost 3 & $9.08 \pm 2.48$ & $47 \pm 14.02$ & $1 \pm 0$ & $0.4 \pm 0.55$ & $51.2 \pm 13.99$ & $0.4 \pm 0.89$ \\
\hline \multirow{8}{*}{ 希 } & 0 & $\mathrm{PI}$ & $11.62 \pm 3.71$ & $57.8 \pm 14.97$ & $1 \pm 1.0$ & $0 \pm 0$ & $37 \pm 15.44$ & $2.8 \pm 0.84$ \\
\hline & 2 & Post-I & $10.32 \pm 2.66$ & $46.5 \pm 7.05$ & $0.7 \pm 0.5$ & $0.25 \pm 0.5$ & $49.5 \pm 7.72$ & $2.75 \pm 0.96$ \\
\hline & 4 & Pre-boost 1 & $10.46 \pm 2.37$ & $44.4 \pm 14.71$ & $0.2 \pm 0.45$ & $0.2 \pm 0.45$ & $53 \pm 14.18$ & $2.2 \pm 0.84$ \\
\hline & 6 & Post-boost 1 & $12.92 \pm 3.27$ & $36.2 \pm 4.44$ & $0.6 \pm 0.55$ & $0 \pm 0$ & $59.8 \pm 4.09$ & $3.4 \pm 1.52$ \\
\hline & 8 & Pre-boost 2 & $12.56 \pm 2.17$ & $51.2 \pm 10.92$ & $0.8 \pm 1.30$ & $0 \pm 0$ & $44.6 \pm 9.94$ & $2.2 \pm 0.84$ \\
\hline & 10 & Post-boost 2 & $18.9 \pm 5.03$ & $30.8 \pm 13.54$ & $0.6 \pm 0.89$ & $0 \pm 0$ & $67.6 \pm 11.93$ & $1 \pm 1.73$ \\
\hline & 12 & Pre-boost 3 & $13.52 \pm 2.53$ & $40.4 \pm 20.44$ & $1 \pm 0.71$ & $0 \pm 0$ & $57.6 \pm 20.01$ & $1 \pm 2.24$ \\
\hline & 15 & Post-boost 3 & $15.54 \pm 4.58$ & $66.2 \pm 5.17$ & $1 \pm 2.24$ & $0 \pm 0$ & $32 \pm 4.53$ & $0.6 \pm 0.55$ \\
\hline \multicolumn{2}{|l|}{$\begin{array}{l}\bar{o} \\
\stackrel{\underline{\nu}}{c} \\
\overline{0}\end{array}$} & Normal range & $6-25.3$ & $21-81$ & $0-6$ & $0-1$ & $17-83$ & $0-5$ \\
\hline
\end{tabular}

${ }^{a}$ CTSOD $n=5$, GPX $n=5$, Control $n=10$.

Values are expressed as mean average $\pm S D$.

Pl, pre-immune; Post-I, post-immune; WBC, total leukocyte count; Neu, neutrophils; Eos, eosinophils; Bas, basophils; Lymp, lymphocytes; Mono, monocytes.

TABLE 2 | Summary of effects of vaccination in baboons (Experiment 1) ${ }^{\text {a }}$.

\begin{tabular}{|c|c|c|c|c|c|c|c|c|}
\hline Week & & Time point & $\operatorname{RBC}\left(\times 10^{6}\right)$ & $\mathrm{Hb}(\mathrm{g} / \mathrm{dl})$ & PVC (\%) & MCV (fl) & MCH (pg) & $\mathrm{MCHC}(\mathrm{g} / \mathrm{dl})$ \\
\hline \multirow{8}{*}{$\begin{array}{l}0 \\
\text { O } \\
\stackrel{5}{0}\end{array}$} & 0 & $\mathrm{Pl}$ & $4.302 \pm 0.3$ & $12.94 \pm 0.93$ & $38.8 \pm 2.77$ & $90.72 \pm 11.5$ & $30.26 \pm 3.83$ & $33.3 \pm 0$ \\
\hline & 2 & Post-I & $4.06 \pm 0.17$ & $12.58 \pm 0.36$ & $33.5 \pm 1.52$ & $82.4 \pm 1.12$ & $30.98 \pm 1.2$ & $37.52 \pm 1.48$ \\
\hline & 4 & Pre-boost 1 & $4.626 \pm 0.29$ & $13.64 \pm 0.15$ & $37.86 \pm 2.48$ & $82.04 \pm 1.66$ & $29.54 \pm 1.91$ & $36 \pm 2.29$ \\
\hline & 6 & Post-boost 1 & $4.166 \pm 0.23$ & $13 \pm 0.19$ & $33.46 \pm 2.69$ & $80.3 \pm 3.46$ & $31.24 \pm 1.81$ & $39.02 \pm 3.55$ \\
\hline & 8 & Pre-boost 2 & $4.398 \pm 0.35$ & $13.56 \pm 0.48$ & $35.42 \pm 4.46$ & $80.34 \pm 4.57$ & $30.94 \pm 2.58$ & $38.7 \pm 4.68$ \\
\hline & 10 & Post-boost 2 & $4.634 \pm 0.19$ & $14.56 \pm 0.21$ & $39.04 \pm 2.47$ & $84.2 \pm 2.37$ & $31.4 \pm 1.63$ & $38.6 \pm 2.58$ \\
\hline & 12 & Pre-boost 3 & $4.248 \pm 0.26$ & $11.94 \pm 1.15$ & $35.42 \pm 2.75$ & $83.32 \pm 3.24$ & $27.92 \pm 2.3$ & $33.54 \pm 3.04$ \\
\hline & 15 & Post-boost 3 & $5.022 \pm 0.19$ & $12.34 \pm 1.1$ & $34.42 \pm 1.20$ & $68.5 \pm 2.96$ & $24.56 \pm 2.12$ & $35.88 \pm 3.82$ \\
\hline \multirow{8}{*}{ 希 } & 0 & $\mathrm{Pl}$ & $4.264 \pm 0.51$ & $13.14 \pm 0.76$ & $40.34 \pm 1.93$ & $95.24 \pm 9.91$ & $31.28 \pm 3.57$ & $33.3 \pm 0$ \\
\hline & 2 & Post-I & $4.59 \pm 0.39$ & $12.77 \pm 0.55$ & $38.25 \pm 3.29$ & $83.35 \pm 0.17$ & $27.9 \pm 2.03$ & $33.55 \pm 2.6$ \\
\hline & 4 & Pre-boost 1 & $4.79 \pm 0.24$ & $14.6 \pm 0.86$ & $39.92 \pm 2.23$ & $83.08 \pm 2.98$ & $31.08 \pm 1.87$ & $36.6 \pm 2.95$ \\
\hline & 6 & Post-boost 1 & $4.532 \pm 0.43$ & $13.78 \pm 0.74$ & $38.36 \pm 6.42$ & $88 \pm 14.44$ & $30.48 \pm 1.46$ & $35.58 \pm 6.35$ \\
\hline & 8 & Pre-boost 2 & $4.596 \pm 0.26$ & $14.64 \pm 0.88$ & $36.48 \pm 2.06$ & $79.36 \pm 0.96$ & $31.8 \pm 0.23$ & $40.08 \pm 0.57$ \\
\hline & 10 & Post-boost 2 & $4.348 \pm 0.23$ & $14.86 \pm 0.96$ & $36.42 \pm 2.25$ & $83.7 \pm 1.46$ & $34.14 \pm 1.32$ & $40.78 \pm 1.99$ \\
\hline & 12 & Pre-boost 3 & $4.554 \pm 0.26$ & $11.92 \pm 0.34$ & $39.82 \pm 4.49$ & $87.14 \pm 4.91$ & $26.18 \pm 1.92$ & $30.12 \pm 3.99$ \\
\hline & 15 & Post-boost 3 & $5.286 \pm 0.52$ & $13.58 \pm 0.89$ & $35.9 \pm 3.23$ & $68.1 \pm 1.81$ & $25.76 \pm 2.17$ & $37.96 \pm 3.51$ \\
\hline \multicolumn{2}{|l|}{$\begin{array}{l}\overline{0} \\
\stackrel{\underline{\nu}}{c} \\
\text { Oे }\end{array}$} & Normal range & $3.64-5.36$ & $11.3-14.5$ & $27.7-49.3$ & 65.4-102 & $23.5-35.2$ & $28.9-44.4$ \\
\hline
\end{tabular}

${ }^{a} \mathrm{CTSOD} n=5 ;$ GPX $n=5$; Control $n=10$.

Values are expressed as mean average $\pm S D$.

PI, pre-immune; Post-I, post-immune; RBC, erythrocyte count; Hb, hemoglobin concentration; PVC, packed cell volume; MCV, mean corpuscular volume; MCH, mean corpuscular hemoglobin; MCHC, mean corpuscular hemoglobin concentration.

baboons each were vaccinated with CTSOD and GPX, as well as ECSOD, and challenged with 600 S. mansoni cercariae. Weekly clinical analysis demonstrated that all three vaccinated groups had higher percentage of animals without diarrhea (CTSOD $=60 \%$, $\mathrm{ECSOD}=60 \%$, and GPX $=40 \%$ ) upon the onset of egg deposition when compared to only $20 \%$ in the Control group (Figure 2B). In addition, animals vaccinated with antioxidants showed fewer mean days of dysentery compared to controls (Figure 2C), where CTSOD group presented with a mean reduction of $75 \%$ (ranging from 100 to $17 \%$ ), ECSOD with 63\% (ranging from 100 to $0 \%$ ), and GPX with 25\% (ranging from 100 to $0 \%$ ).

The weekly assessment of the EPG commencing 6 weeks after challenge showed that vaccinated groups had an overall reduction of eggs when compared to the Control group (Figures 3A-F). This reduction was statistically significant within ECSOD at week 8 (Figure 3C) when compared to the unvaccinated Control group. When the total EPG over a 5-week period was analyzed, all vaccinated groups showed reduction of excreted eggs, specially 

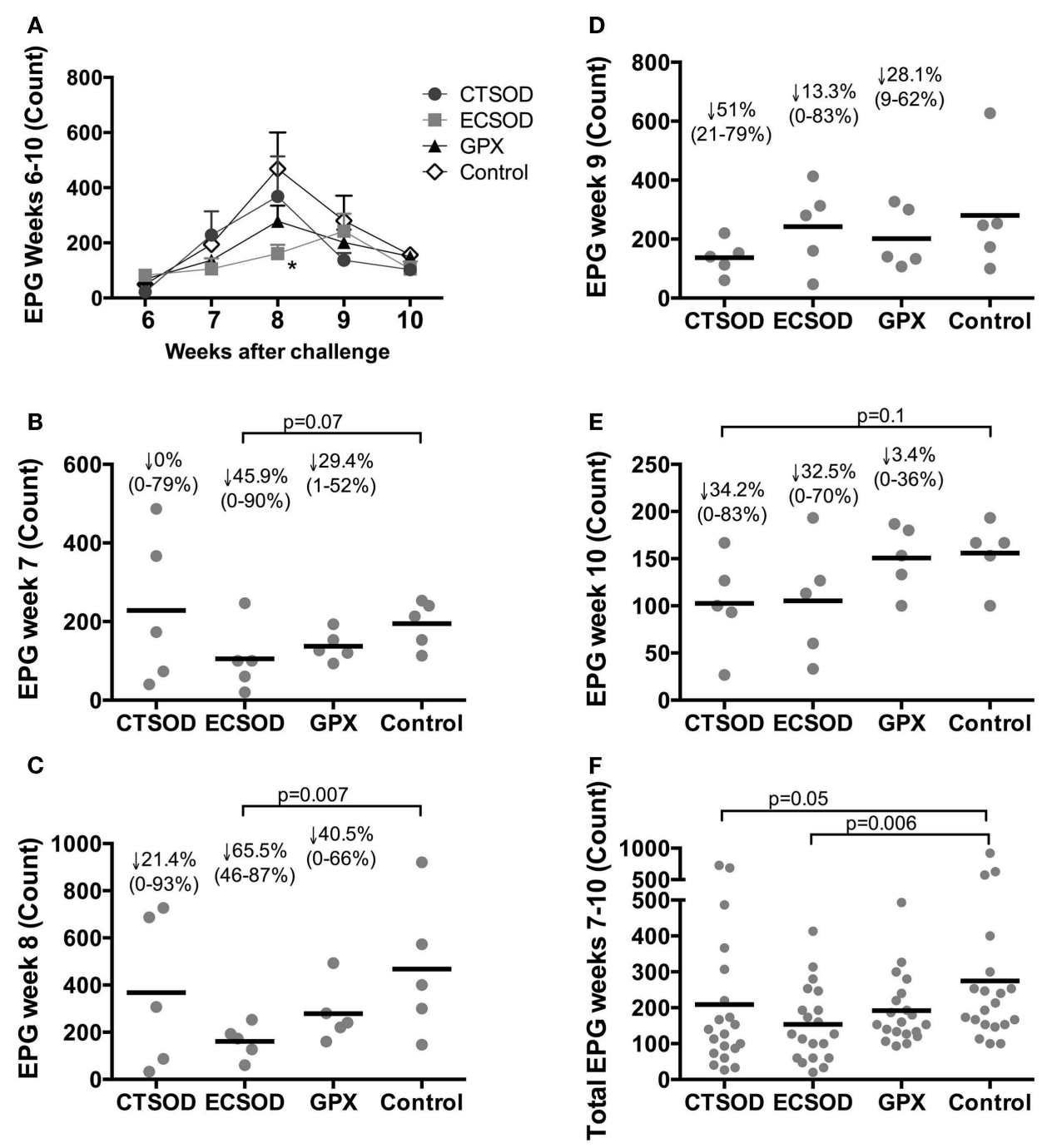

FIGURE 3 | Dynamics of weekly egg excretion per gram of feces (EPG) from Experiment 2. Assessment of eggs per gram of feces (EPG) from vaccinated and control baboons ( $n=5$ animals per group) commencing 6 weeks after S. mansoni cercariae challenge: weekly overview (A); week 7 (B); week 8 (C); week 9 (D); week 10 (E); and the total EPG

excreted over 5 weeks period (F). The statistical $p$-value was expressed in comparison to the mean average of the Control group. Results were log-transformed and corrected for multiple analyses when applying ANOVA and student's $t$-tests. Values were considered statistically significant when $p<0.05$.

ECSOD vaccinated animals who showed statistically significant reduction when compared to the Control (Figure 3F).

\section{Tissue Eggs from Liver, Small Gut, and Large Gut (Experiment 2)}

The distribution of eggs recovered in harvested organs after $\mathrm{KOH}$ digestion from vaccinated and control baboons 10 weeks after challenge at perfusion time point is shown in Figure 4. There was an overall reduction of eggs in all vaccinated groups in the liver, small gut, and large gut when compared to the Control group. The egg reduction in the liver (Figure 4A) ranged from 56 to $0 \%$ for CTSOD, 84 to 3\% for ECSOD, and 56 to $0 \%$ for GPX, while in the small gut (Figure 4B), the reduction in CTSOD group ranged from 64 to $0 \%$, ECSOD ranged from 88 to $0 \%$, and GPX ranged from 100 to $0 \%$. When the large gut was evaluated (Figure 4C), egg reduction ranged from 70 to $0 \%$ for CTSOD, 93 to $0 \%$ for ECSOD, and 89 to $0 \%$ for GPX, when compared to the mean average of Control group.

\section{Worm Burden/Percentage of Protection (Experiment 2)}

When the distribution of worms (Figure 5A), males (Figure 5B), and females (Figure 5C) recovered from outbred vaccinated and control baboons 10 weeks after challenge with S. mansoni cercariae was evaluated at the perfusion time point, ECSOD vaccinated group showed a reduction of total worms that ranged from 89 to $0 \%$ (mean protection $19.5 \%$ ), while GPX group showed a reduction that ranged from 47 to $0 \%$ (mean protection $17.1 \%$ ) 


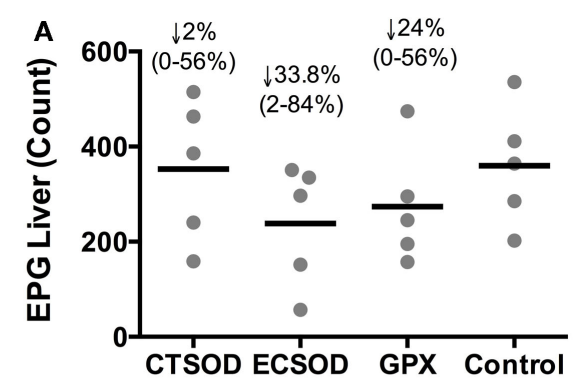

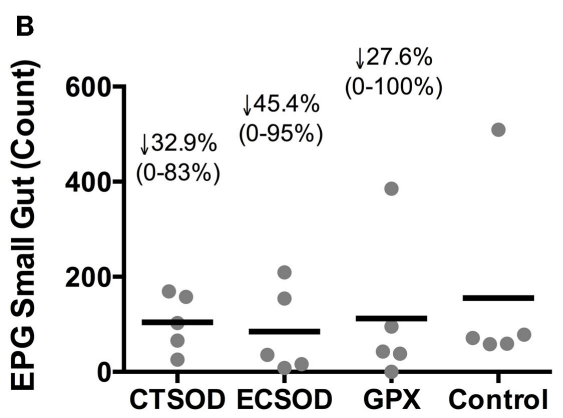

FIGURE 4 | Distribution of eggs recovered in tissues from Experiment 2. Liver (A), small gut (B), and large gut (C) eggs after $\mathrm{KOH}$ digestion from vaccinated and control baboons ( $n=5$ animals per group) 0 weeks after challenge with $S$. mansoni cercariae (perfusion time point). Individual values (sphere); mean average (dash). The percentage of

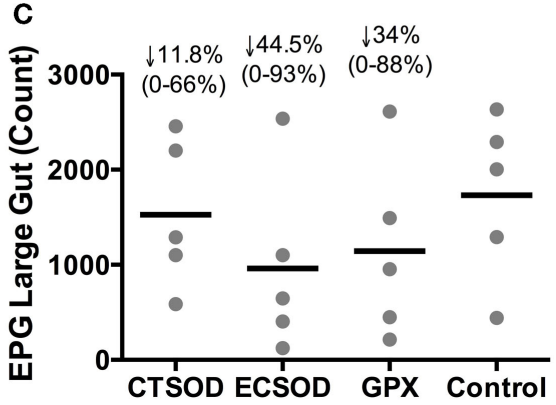

reduction (represented by the arrow), the range (minimal-maximal, represented between brackets), and the statistical $p$-value were expressed in comparison to the mean average of the Control group. Non-parametric (Kruskal-Wallis, Mann-Whitney) tests were applied. Values were considered statistically significant when $p<0.05$.
B

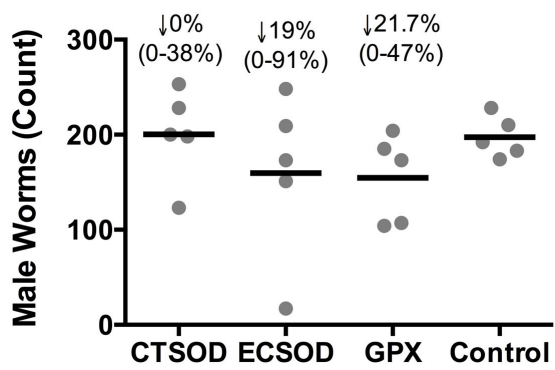

FIGURE 5 | Distribution of worms recovered (worm burden) from Experiment 2. Total worm burden (A), male (B) and female (C) worm count. Worms from vaccinated and control baboons $(n=5$ animals per group) 10 weeks after challenge with S. mansoni cercariae (perfusion time point). Individual values (sphere); mean average (dash). The

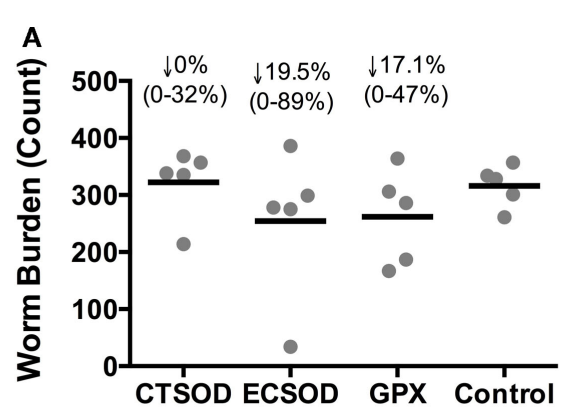

C

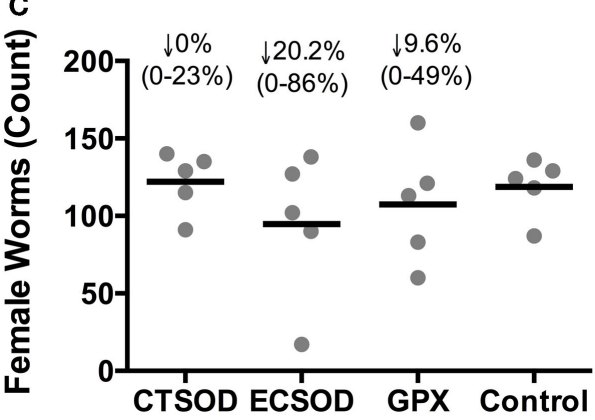

percentage of reduction (represented by the arrow), the range (minimalmaximal, represented between brackets), and the statistical $p$-value were expressed in comparison to the mean average of the Control group. Nonparametric (Kruskal-Wallis, Mann-Whitney) tests were applied. Values were considered statistically significant when $p<0.05$. 
of worms when compared to the Control group (Figure 5A). The same pattern was observed when worms were stratified into males (Figure 5B) and females (Figure 5C). The CTSOD group failed to show a mean reduction in worms, although reduction in two vaccinated baboons (out of five) is visible.

\section{Antibody Response (Experiment 2)}

Titration of baboon sera against recombinant antigens showed that levels of specific IgG antibodies were stimulated after immunizations (PC) with CTSOD (Figure 6A) as well as with GPX (Figure 6D), and to a lesser degree with ECSOD (Figure 6B), when compared to control sera (Figures 6C,E). Antibody levels continued to be elevated 10 weeks after challenge infection (PP) for CTSOD and GPX groups.

\section{Proliferation/Recall Responses (Experiment 2)}

Recall proliferation assays from PBMCs (Figures 7A-C) were performed against crude and recombinant antigens before (preimmune, PI), after immunizations (pre-challenge, PC), and after challenge with $S$. mansoni at the perfusion time point (PP). The recall proliferation assays for mesenteric (MLN) and inguinal (ILN) lymph nodes (Figures 7D-E) were performed only at the time of the perfusion (PP). All results were expressed as Stimulation Index (S.I.): the ratio of the mean cpm of triplicate culture cells taken up in the presence of the antigen over those obtained with medium alone. PBMC (Figures 7A,B) and MLN cells (Figures 7D,E) from both CTSOD and ECSOD groups proliferated in response to recombinant CTSOD antigens when compared with Control. Similarly, PBMC (Figure 7C), mesenteric, and inguinal node
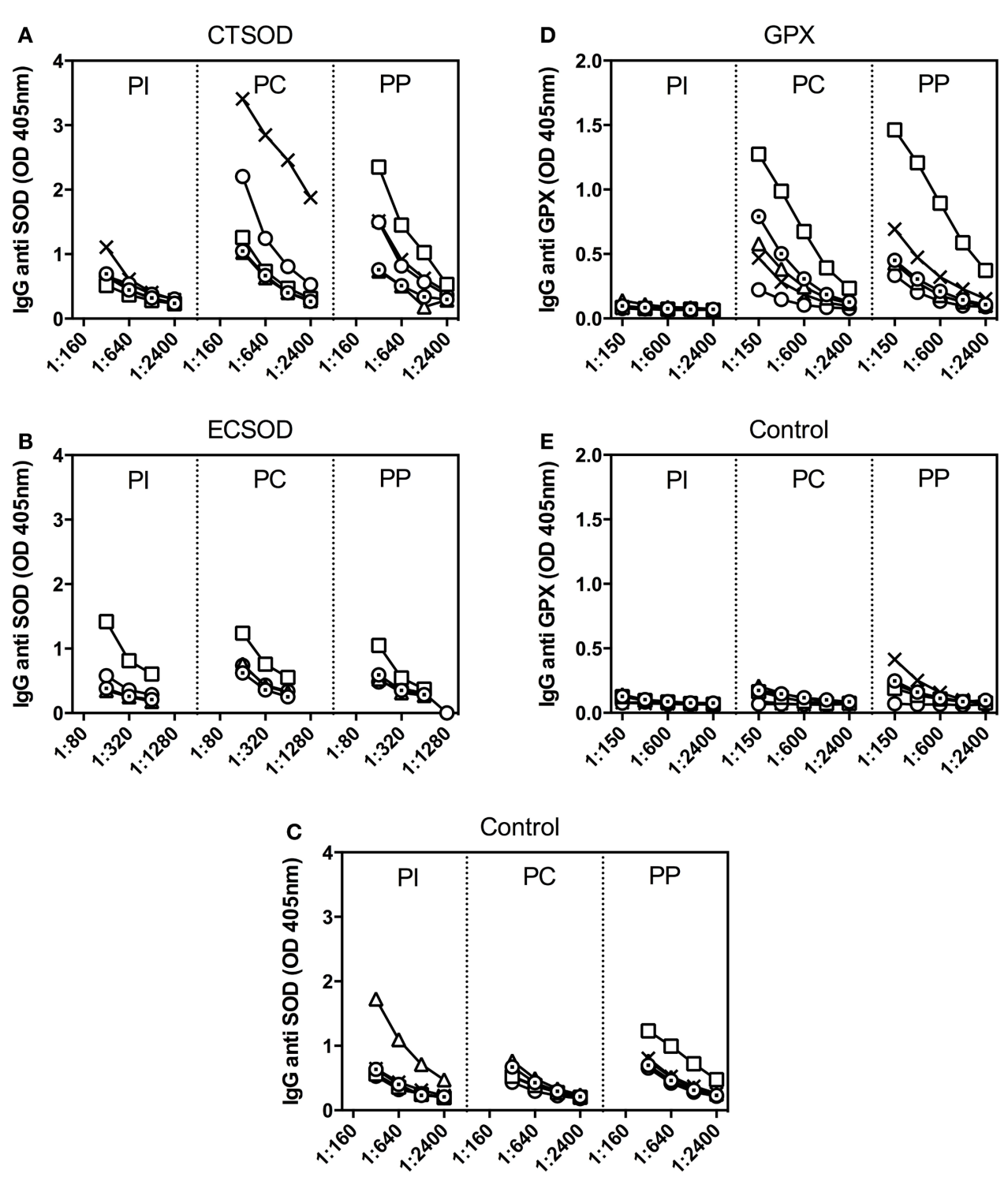

FIGURE 6 | Antibody response from Experiment 2. Titration of baboon lgG antibodies against recombinant SOD (A-C) and GPX (D-E) from CTSOD (A), ECSOD (B), GPX (D), and Control (C,E) groups ( $n=5$ animals per group) before (PI), after immunizations (pre-challenge, PC), and before perfusion (pre-perfusion, PP). 

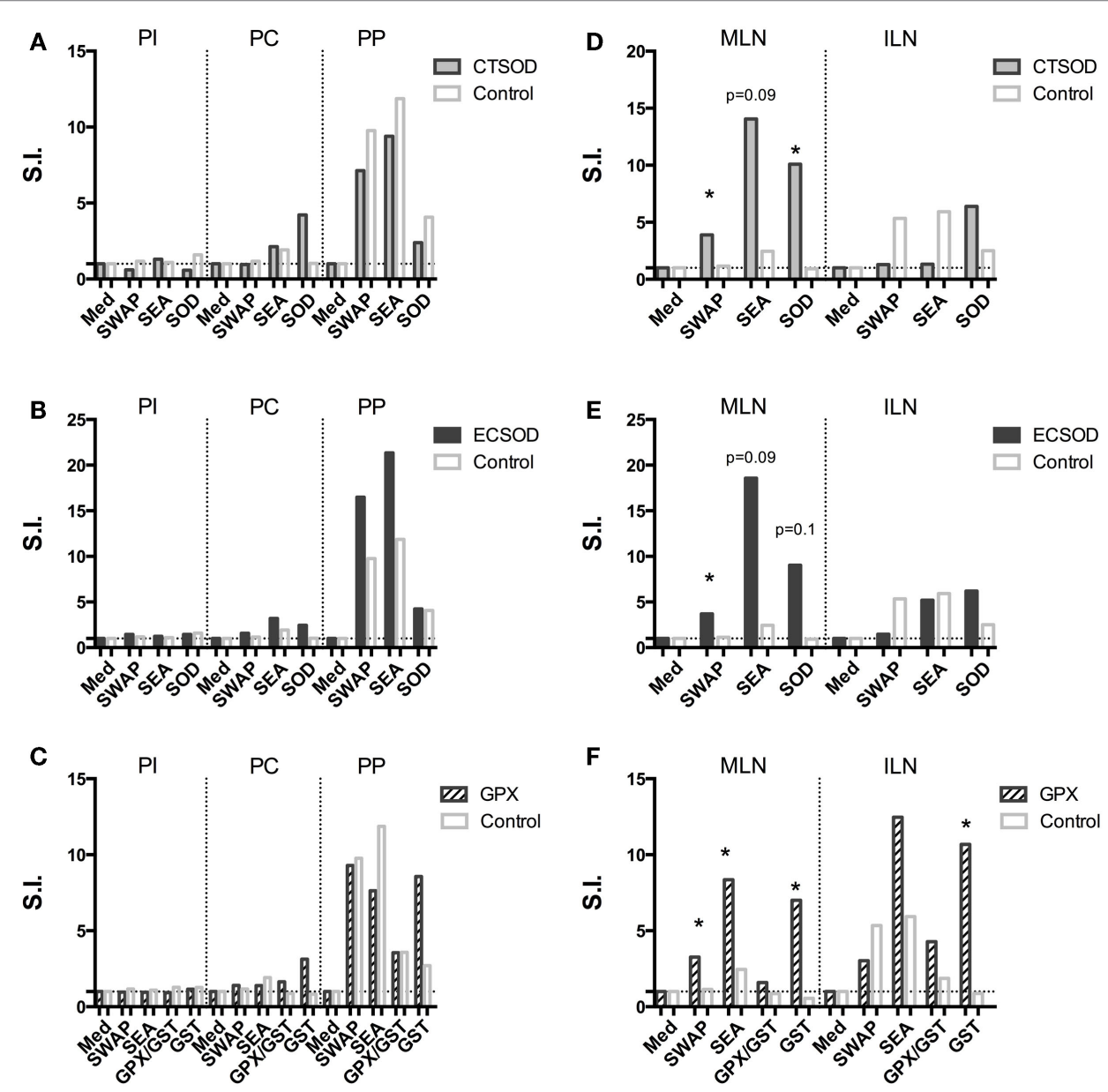

FIGURE 7 | Recall proliferation assays from Experiment 2. PBMC (A-C) and mesenteric (MLN) and inguinal (ILN) lymph nodes (D-F) against crude and recombinant antigens from CTSOD (A,D), ECSOD (B,E), GPX (C,F), and Control groups ( $n=5$ animals per group) before (PI), after immunizations (pre-challenge, $\mathrm{PC}$ ), and after challenge with $\mathrm{S}$. mansoni at the perfusion time

point (PP). Results were expressed as Stimulation Index (S.I.), the ratio of the mean counts per minute (cpm) of triplicate culture cells taken up in the presence of the antigen over those obtained with medium alone. Nonparametric (Kruskal-Wallis, Mann-Whitney) tests were applied. Values were considered statistically significant when $p<0.05$.

cells (Figure 7F) from GPX vaccinated animals proliferated in response to recombinant antigens in comparison with Control group. PBMC and MLN from all groups responded to SEA and SWAP after challenge with S. mansoni cercariae (Figures 7D-F).

\section{Secretion of Cytokines and Chemokines upon Stimulation (Experiment 2)}

A panel of 23 multiplexed IFN- $\gamma$, IL-12, IL-4, IL-6, IL-17A, CCL3 (MIP-1 $\alpha$ ), IL-5, IL-13, CCL2 (MCP-1), IL-1 $\beta$, IL-2, IL-15, CCL4 (MIP-1 $\beta$ ), TNF- $\alpha$, IL-10, TGF- $\alpha$, IL-1Ra, IL-8, GM-CSF, sCD40L, VEGF, G-CSF, IL-18 cytokines and chemokines (Figures 8-11; Figures S1 and S2 in Supplementary Material) was used to investigate secretion from individual supernatants derived from PBMCs from CTSOD (A, D, G), ECSOD (B, E, H), and GPX (C, F, I) groups after $72 \mathrm{~h}$ stimulation with crude and recombinant antigens at all time points: before vaccination (PI), before challenge (PC), and at perfusion (PP). Values were expressed as median of cytokine secretion from five individual supernatants.
In general, vaccination with ECSOD and CTSOD induced variable levels of cytokines before challenge (PC) upon stimulation with recombinant antigens when compared to the baseline levels (PI), although these levels were somewhat discrete. However, vaccination with GPX induced higher levels of IL-5 (Figure 8F) and IL-17A (Figure 9I) cytokines, and reduction of IL-8 (Figure 10F) and chemokines such as CCL2, CCL3, and CCL4 (Figures 11C,F,I) when compared to Control. CTSOD vaccination induced increase in the IL-17A levels (Figure 9G), while a reduction of IL-1 $\beta$, IL-18 (Figures 10A,B,G,H) cytokines, and CCL3 and CCL4 (Figures 11C,I) chemokines in CTSOD and ECSOD groups was also observed, when compared to Control.

After challenge, several cytokine and chemokine levels increased in the vaccinated groups after the natural boost provided from the infection with $S$. mansoni (Figures 8-11). For instance, high levels of IL-4, IL-5, IL-13 (Figures 8A-I), IFN- $\gamma$ (Figures 9A-C) cytokines were stimulated after challenge (PP) against SWAP and SEA in the CTSOD, ECSOD, and GPX groups when compared 

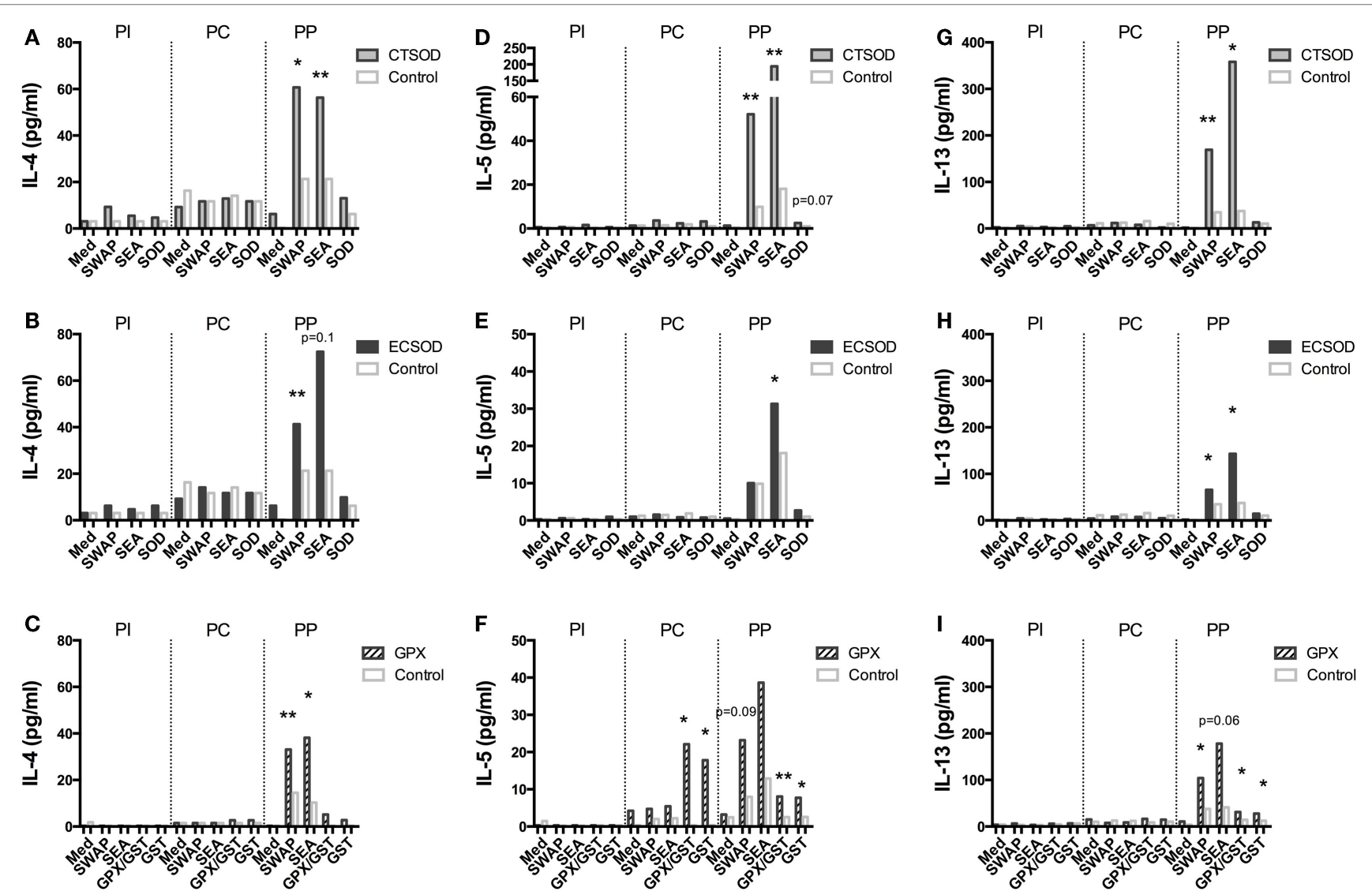

FIGURE 8 | Secretion of cytokines in supernatants from Experiment 2. PBMCs from CTSOD (A,D, G), ECSOD (B,E,H), and GPX (C, F, I) groups ( $n=5$ animals per group) were stimulated $72 \mathrm{~h}$ with crude and recombinant antigens and secreted IL-4, IL-5, and IL-13 levels determined, before vaccination $(\mathrm{PI})$, before challenge $(\mathrm{PC})$, and at perfusion $(\mathrm{PP})$. Values were expressed as median of cytokine secretion in five individual supernatants in relation to the Control baboons. Non-parametric (Kruskal-Wallis, MannWhitney) tests were applied. Values were considered statistically significant when $p<0.05$, and assigned ${ }^{*} p=0.01-0.05 ;{ }^{* *} p=0.001-0.01$; and ${ }^{\star \star \star} p=<0.001$. to (PC) levels, and this response was higher in the vaccinated groups when compared to the control group. The levels of IL-15 (Figures 9D-F) were also increased, especially in the GPX group, before perfusion (PP) when compared to control.

\section{Discussion}

As mentioned previously, our laboratory has demonstrated that vaccination of inbred mice with naked DNA constructs containing $\mathrm{Cu} / \mathrm{Zn}$ cytosolic superoxide dismutase (CT-SOD), signal-peptide containing SOD (EC-SOD), or glutathione peroxidase (GPX) derived from $S$. mansoni showed significant levels of protection compared to a control group (29). Screening of candidate antigens in mice is an important first step in vaccine development but it is unclear whether protective efficacy can be translated directly to humans (38). Therefore, our murine results encouraged us to investigate if immunization of non-human primates with these antioxidants would stimulate an immune response that could be correlated with protection as a prelude study for human trials. Baboons are an excellent model for schistosomiasis, as they are similar to humans in ontogeny, immune response (including human-like IgG subclasses), reproductive physiology, etc., and develop a human-like acute disease after natural or experimental exposure to S. mansoni (38-40). In addition, since issues of vaccine safety are also difficult to address in mice, another advantage of testing schistosome vaccines in baboons is the opportunity to address many of the deficiencies of mouse studies (38-41). We have also previously used baboons to successfully investigate the immune mechanisms associated with other schistosome vaccine candidates (42). Overall, our data indicate that no adverse reactions or abnormal animal demeanor occurred to the immunization of baboons with antioxidants, as our behavioral, clinical, and hematological evaluations of the vaccinated animals remained within the normal limits throughout the observation period for both experiments. Thus the vaccines are safe and well tolerated.

The next step was to check the antigenicity of the antioxidants after vaccination with baboons, as the dose and nature of the antigen as well as the route or kind of adjuvant used can ultimately dictate the outcome of an immunization, among other factors. Plasmid/ naked DNA vaccines are less toxic, but also less immunogenic, therefore potent and safe adjuvants that can be used as vaccine delivery systems and as immunostimulatory adjuvants are necessary (43). Biodegradable and biocompatible polyester polymers such as PLA to encapsulate antigens (44-46) have been successfully 

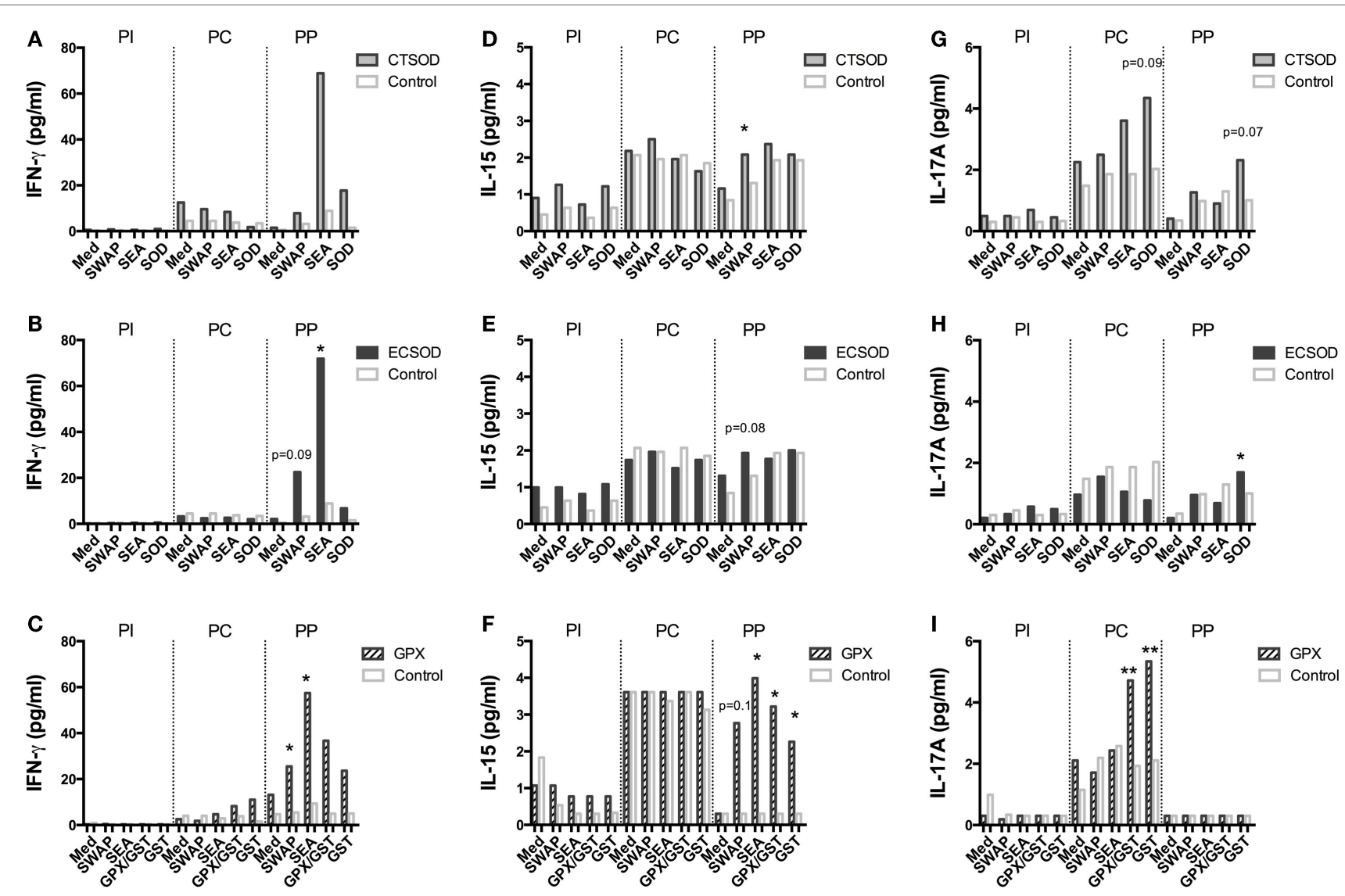

FIGURE 9 | Secretion of cytokines in supernatants from Experiment 2 PBMCs from CTSOD (A,D,G), ECSOD (B,E,H), and GPX (C, F, I) groups ( $n=5$ animals per group) were stimulated $72 \mathrm{~h}$ with crude and recombinant antigens and secreted IFN- $\gamma$, IL-15, and IL-17A levels determined, before vaccination $(\mathrm{PI})$, before challenge $(\mathrm{PC})$, and at perfusion (PP). Values were expressed as median of cytokine secretion in five individual supernatants in relation to the Control baboons. Non-parametric (Kruskal-Wallis, MannWhitney) tests were applied. Values were considered statistically significant when $p<0.05$, and assigned ${ }^{*} p=0.01-0.05 ;{ }^{*} p=0.001-0.01$; and ${ }^{* * *} p=<0.001$. used in applications ranging from cancer therapy to infectious diseases (47-50). The enhanced adjuvant effect of such microparticles appears to be a consequence of efficient and controlled delivery of the adsorbed proteins into dendritic cells and macrophages at the injection site and local lymph nodes $(51,52)$. In the murine model, we showed that along with DNA immunization, one single dose of SmCT-SOD proteins encapsulated in PLA microspheres, for instance, was able to induce high titers of specific antibodies in immunized mice (32). Therefore, in our baboon studies, we evaluated if the immunization protocol of priming with naked DNA and boosting with the respective antioxidant proteins encapsulated in PLA microspheres could meet or even enhance the immunogenicity and protection levels achieved by prime-boost immunization with DNA only. All three antioxidant vaccines were immunogenic to different degrees, stimulating both humoral and cellular responses (including cytokines and chemokines). As observed previously by us and by other studies, the specific antibody and cellular responses primed by DNA vaccination were boosted by infection. This natural boosting is believed to be beneficial in endemic areas where individuals are continuously exposed to the parasite $(31,53,54)$.
Numerous studies on individuals in endemic areas for Schistosoma showed that parasite-specific humoral and cellular responses vary in their correlation with the development of resistance and/or susceptibility/morbidity to infection/re-infection (55-57). And an increasing body of evidence indicates that a balance between innate cells and CD4+ T helper (Th) cells Th1, Th2, Th17, and Treg responses (which cross-regulate one another during infection) rather than a polarizing effect (e.g., Th1 vs Th2) is likely beneficial in the development of protection against $S$. mansoni infections, both in humans and experimental models (58-61). Moreover, it is becoming evident that the interaction between these responses rather than just the levels of individual cytokines alone may influence outcomes such as resistance to reinfection $(62,63)$.

In our study, a mixed cytokine/chemokine response was observed, where the $S$. mansoni infection stimulated a Th2 response in all groups as well as an inflammatory profile that differed from controls. Although it was clear that vaccination with ECSOD, CTSOD, and GPX stimulated different responses when compared to the control group. The specific role of antibodies or cellular responses and the development of protection against 

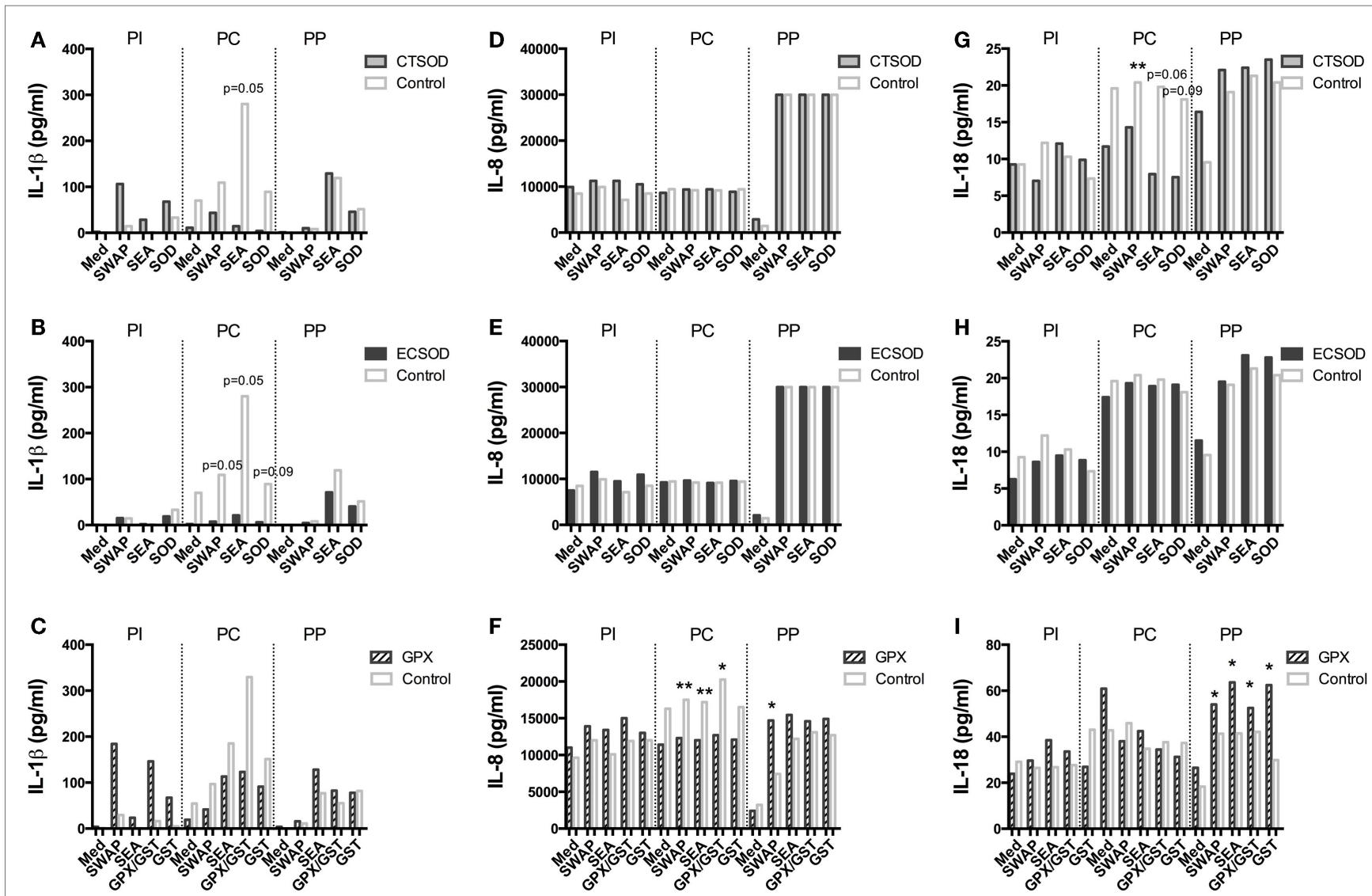

FIGURE 10 | Secretion of cytokines in supernatants from Experiment 2. PBMCs from CTSOD (A,D,G), ECSOD (B,E,H), and GPX (C, F,I) groups ( $n=5$ animals per group) were stimulated $72 \mathrm{~h}$ with crude and recombinant antigens and secreted IL-1 $\beta$, IL-8, and IL-18 levels determined, before vaccination $(\mathrm{PI})$, before challenge $(\mathrm{PC})$, and at perfusion (PP). Values were expressed as median of cytokine secretion in five individual supernatants in relation to the Control baboons. Non-parametric (Kruskal-Wallis, MannWhitney) tests were applied. Values were considered statistically significant when $p<0.05$, and assigned ${ }^{*} p=0.01-0.05 ;{ }^{* *} p=0.001-0.01$; and ${ }^{\star \star *} p=<0.001$. challenge with S. mansoni is still unknown, and currently under investigation. As shown by Mola et al. (64), the levels of most cytokines produced by PBMC or antibodies vary depending on whether the baboons experienced a primary infection compared to re-infection with S. mansoni. Although the young baboons used in this study were caught in the wild from non-endemic areas and were quarantined and checked for the presence of current schistosomiasis infection (and other infections), we cannot rule out the possibility that some of these animals were infected prior to the beginning of our study, therefore influencing the cytokine responses. However, this in fact would most likely resemble the situation in endemic areas, where people in endemic areas would be exposed to one or multiple infections or none at all before vaccination with antioxidants, and in many instances, such exposure to worm antigens in humans would have occurred very early in life during childhood or even in utero $(65,66)$, which makes outbred baboons ideally suited for such studies.

In schistosomiasis, pathogenesis is mainly caused by the immunological reaction of the host to the eggs (and their secretions) released by adult worm pairs that inhabit the portal circulation. Consequently, the severity of the disease is directly related to the worm burden and the inflammatory response to deposited eggs (67). For instance, as recently reviewed (68), the chemokines CCL2, CCL3, and CCL4 correlate with the severity of S. mansoni, where CCL3 is related to the recruitment of eosinophils and induction of granulomatous pathology (69), while CCL2 is associated with glomerulopathy (70). A more "traditional" vaccine concept would target a reduction of the worm number or even prevent infection. However, since the released eggs are responsible for both transmission and pathology, a vaccine targeted at parasite fecundity and egg viability has become relevant $(71,72)$. The vaccination of baboons with antioxidants in the present study stimulated a partial reduction in the worm burden, which was in sharp contrast with our previous studies in mice (29). However, vaccination with antioxidants, especially ECSOD, promoted a strong anti-fecundity and anti-pathology effect, by means of an overall reduction of eggs in all vaccinated groups in the feces, liver, small gut, and large gut, as well as reduction of diarrhea (dysentery) when compared to the Control group. One of the hallmarks of pathology, diarrhea normally occurs during moderate and heavy $S$. mansoni infections due to eggs released by the worms, which in turn induce an immunological response leading to pathology 

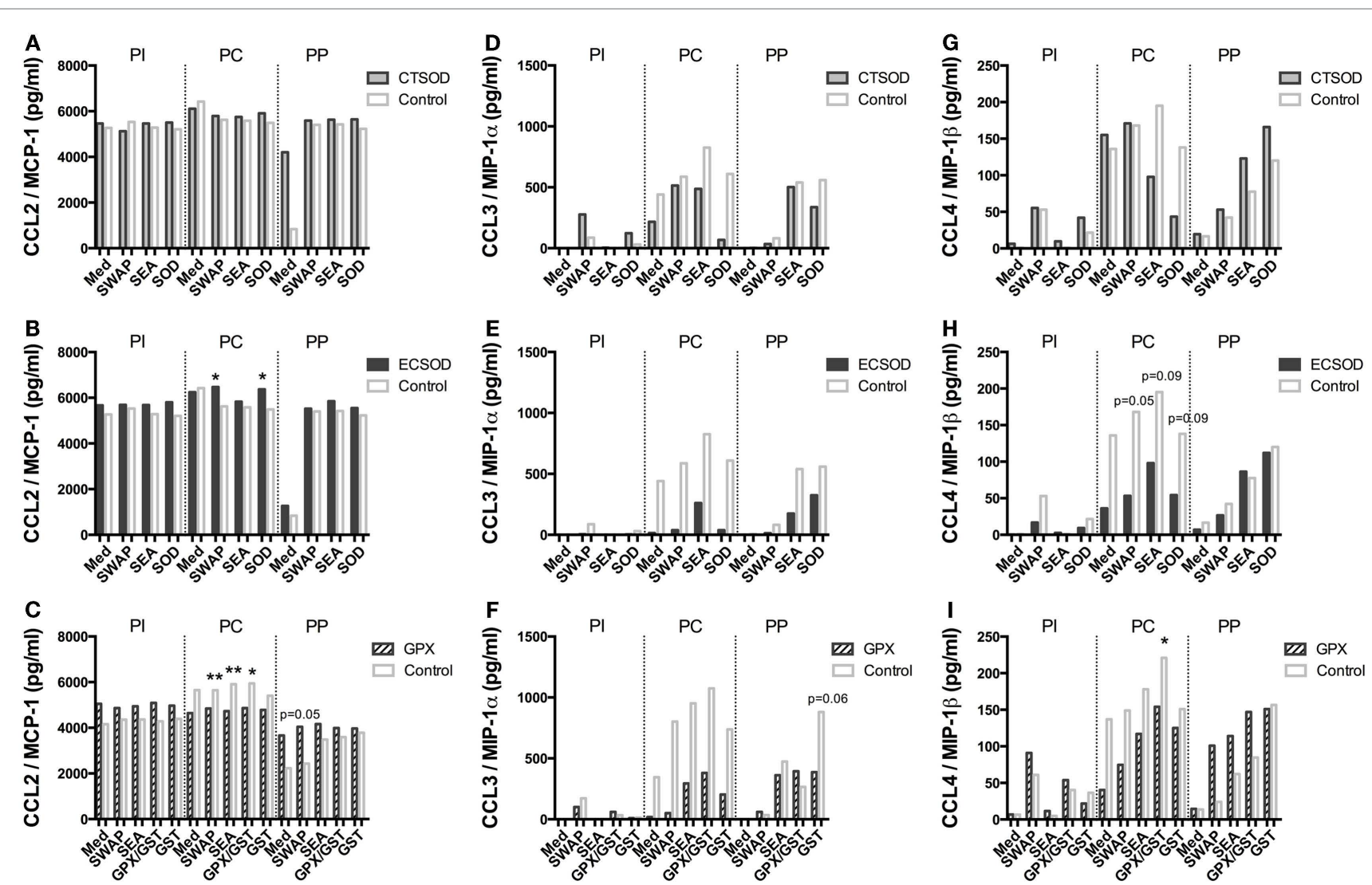

FIGURE 11 | Secretion of chemokines in supernatants from Experiment 2. PBMCs from CTSOD (A,D,G), ECSOD (B,E, $\mathbf{H})$, and GPX (C, $\mathbf{C}, \mathbf{I})$ groups ( $n=5$ animals per group) were stimulated $72 \mathrm{~h}$ with crude and recombinant antigens and secreted CCL2 (MCP-1), CCL3 (MIP-1 $\alpha$ ), and CCL4 (MIP-1 $\beta$ ) levels determined, before vaccination $(\mathrm{PI})$, before challenge $(\mathrm{PC})$, and at perfusion (PP). Values were expressed as median of cytokine secretion in five individual supernatants in relation to the Control baboons. Non-parametric (Kruskal-Wallis, Mann-Whitney) tests were applied. Values were considered statistically significant when $p<0.05$, and assigned ${ }^{*} p=0.01-0.05$;

${ }^{\star *} p=0.001-0.01 ;$ and ${ }^{* \star *} p=<0.001$. in the intestine wall (67). In our study, diarrhea was reduced in all vaccinated groups, in particular in the CTSOD and ECSOD groups. Other studies had reported both immune responses and anti-fecundity effects against female worms with glutathione-Stransferase, $\operatorname{SmGST}(73,74)$, as well as the large subunit of calpain, Sm-p80 (75). Interestingly, the significant reduction in fecundity in this study was in spite of the small reduction in worm burden. The mechanism of the anti-fecundity effect by antioxidants or any other vaccine candidate, which seems to be independent of worm burden, remains to be determined.

Taken together, our results challenge the common concept for markers and correlates of protection, since in despite of the lower than $40 \%$ worm reduction, as set by WHO, the high anti-fecundity/ anti-pathology effect observed by means of reduction of eggs and overall pathology indicates that these antioxidants vaccines could prevent intestinal pathology and therefore warrants further investigation. In addition, the use of outbred juvenile baboons more accurately reflects the human situation as regards response to vaccination and subsequent challenge infection. Experiments with different vaccine regimens (including multivalent immunizations), delivery modes, and adjuvants in the baboon model are planned for the near future.

\section{Acknowledgments}

We would like to thank Gregory Sonnenberg for the excellent assistance in preparing GPX recombinant proteins. We also would like to thank the excellent technical support of Fred Nyundo, Simon Kiarie, and Sammy Kisara who assisted with parasite assays. We also thank Habib Saibulu, James Ndungu, Zachariah Maheli, and the team from Animal Sciences at IPR, who routinely offered professional husbandry, veterinary, and surgical care to the study animals as required. This research was supported by NIAID grant AI18867.

\section{Supplementary Material}

The Supplementary Material for this article can be found online at http://journal.frontiersin.org/article/10.3389/fimmu.2015.00273/ abstract 


\section{References}

1. Engels D, Chitsulo L, Montresor A, Savioli L. The global epidemiological situation of schistosomiasis and new approaches to control and research. Acta Trop (2002) 82(2):139-46. doi:10.1016/S0001-706X(02)00045-1

2. Hotez PJ, Fenwick A, Savioli L, Molyneux DH. Rescuing the bottom billion through control of neglected tropical diseases. Lancet (2009) 373(9674):1570-5. doi:10.1016/S0140-6736(09)60233-6

3. Hotez PJ, Molyneux DH, Fenwick A, Kumaresan J, Sachs SE, Sachs JD, et al. Control of neglected tropical diseases. N Engl J Med (2007) 357(10):1018-27. doi:10.1056/NEJMra064142

4. Kirby T. David Molyneux: raising the profile of neglected tropical diseases. Lancet (2010) 375(9708):21. doi:10.1016/S0140-6736(09)62174-7

5. Fenwick A, Webster JP. Schistosomiasis: challenges for control, treatment and drug resistance. Curr Opin Infect Dis (2006) 19(6):577-82. doi:10.1097/01. qco.0000247591.13671.6a

6. Fenwick A, Webster JP, Bosque-Oliva E, Blair L, Fleming FM, Zhang Y, et al. The schistosomiasis control initiative (SCI): rationale, development and implementation from 2002-2008. Parasitology (2009) 136(13):1719-30. doi:10.1017/ S0031182009990400

7. Kabatereine NB, Brooker S, Koukounari A, Kazibwe F, Tukahebwa EM, Fleming FM, et al. Impact of a national helminth control programme on infection and morbidity in Ugandan schoolchildren. Bull World Health Organ (2007) 85(2):91-9. doi:10.2471/BLT.06.030353

8. Kabatereine NB, Tukahebwa E, Kazibwe F, Namwangye H, Zaramba S, Brooker $\mathrm{S}$, et al. Progress towards countrywide control of schistosomiasis and soil-transmitted helminthiasis in Uganda. Trans R Soc Trop Med Hyg (2006) 100(3):208-15. doi:10.1016/j.trstmh.2005.03.015

9. Bergquist NR, Leonardo LR, Mitchell GF. Vaccine-linked chemotherapy: can schistosomiasis control benefit from an integrated approach? Trends Parasitol (2005) 21(3):112-7. doi:10.1016/j.pt.2005.01.001

10. Bergquist R, Johansen MV, Utzinger J. Diagnostic dilemmas in helminthology: what tools to use and when? Trends Parasitol (2009) 25(4):151-6. doi:10.1016/j. pt.2009.01.004

11. Bergquist R, Utzinger J, McManus DP. Trick or treat: the role of vaccines in integrated schistosomiasis control. PLoS Negl Trop Dis (2008) 2(6):e244. doi:10.1371/ journal.pntd.0000244

12. Utzinger J, Bergquist R, Shu-Hua X, Singer BH, Tanner M. Sustainable schistosomiasis control - the way forward. Lancet (2003) 362(9399):1932-4. doi:10.1016/ S0140-6736(03)14968-9

13. Bennett JL, Day T, Liang FT, Ismail M, Farghaly A. The development of resistance to anthelmintics: a perspective with an emphasis on the antischistosomal drug praziquantel. Exp Parasitol (1997) 87(3):260-7. doi:10.1006/expr.1997.4229

14. Fallon PG, Sturrock RF, Niang AC, Doenhoff MJ. Short report: diminished susceptibility to praziquantel in a Senegal isolate of Schistosoma mansoni. Am J Trop Med Hyg (1995) 53(1):61-2.

15. Koukounari A, Fenwick A, Whawell S, Kabatereine NB, Kazibwe F, Tukahebwa EM, et al. Morbidity indicators of Schistosoma mansoni: relationship between infection and anemia in Ugandan schoolchildren before and after praziquantel and albendazole chemotherapy. Am J Trop Med Hyg (2006) 75(2):278-86.

16. Leenstra T, Coutinho HM, Acosta LP, Langdon GC, Su L, Olveda RM, et al. Schistosoma japonicum reinfection after praziquantel treatment causes anemia associated with inflammation. Infect Immun (2006) 74(11):6398-407. doi:10.1128/ IAI.00757-06

17. Olveda RM, Daniel BL, Ramirez BD, Aligui GD, Acosta LP, Fevidal P, et al. Schistosomiasis japonica in the Philippines: the long-term impact of population-based chemotherapy on infection, transmission, and morbidity. J Infect Dis (1996) 174(1):163-72. doi:10.1093/infdis/174.1.163

18. Steinmann P, Keiser J, Bos R, Tanner M, Utzinger J. Schistosomiasis and water resources development: systematic review, meta-analysis, and estimates of people at risk. Lancet Infect Dis (2006) 6(7):411-25. doi:10.1016/S1473-3099(06)70521-7

19. King $\mathrm{CH}$, Dangerfield-Cha $\mathrm{M}$. The unacknowledged impact of chronic schistosomiasis. Chronic Illn (2008) 4(1):65-79. doi:10.1177/1742395307084407

20. McManus DP, Loukas A. Current status of vaccines for schistosomiasis. Clin Microbiol Rev (2008) 21(1):225-42. doi:10.1128/CMR.00046-07

21. Wilson RA, Coulson PS. Schistosome vaccines: a critical appraisal. Mem Inst Oswaldo Cruz (2006) 101(Suppl 1):13-20. doi:10.1590/S0074-02762006000900004
22. Huang HH, Rigouin C, Williams DL. The redox biology of schistosome parasites and applications for drug development. Curr Pharm Des (2012) 18(24):3595-611.

23. Loverde PT. Do antioxidants play a role in schistosome host-parasite interactions? Parasitol Today (1998) 14(7):284-9. doi:10.1016/S0169-4758(98)01261-7

24. Mei H, Thakur A, Schwartz J, Lo Verde PT. Expression and characterization of glutathione peroxidase activity in the human blood fluke Schistosoma mansoni. Infect Immun (1996) 64(10):4299-306.

25. Mei H, LoVerde PT. Schistosoma mansoni: the developmental regulation and immunolocalization of antioxidant enzymes. Exp Parasitol (1997) 86(1):69-78. doi:10.1006/expr.1997.4150

26. La Flamme AC, Patton EA, Bauman B, Pearce EJ. IL-4 plays a crucial role in regulating oxidative damage in the liver during schistosomiasis. JImmunol (2001) 166(3):1903-11. doi:10.4049/jimmunol.166.3.1903

27. Hong Z, Kosman DJ, Thakur A, Rekosh D, LoVerde PT. Identification and purification of a second form of $\mathrm{Cu} / \mathrm{Zn}$ superoxide dismutase from Schistosoma mansoni. Infect Immun (1992) 60(9):3641-51.

28. Maizels RM, Bundy DA, Selkirk ME, Smith DF, Anderson RM. Immunological modulation and evasion by helminth parasites in human populations. Nature (1993) 365(6449):797-805. doi:10.1038/365797a0

29. Shalaby KA, Yin L, Thakur A, Christen L, Niles EG, LoVerde PT. Protection against Schistosoma mansoni utilizing DNA vaccination with genes encoding $\mathrm{Cu} / \mathrm{Zn}$ cytosolic superoxide dismutase, signal peptide-containing superoxide dismutase and glutathione peroxidase enzymes. Vaccine (2003) 22(1):130-6. doi:10.1016/ S0264-410X(03)00535-8

30. Bergquist NR. Controlling schistosomiasis by vaccination: a realistic option? Parasitol Today (1995) 11(5):191-4. doi:10.1016/0169-4758(95)80157-X

31. Cook RM, Carvalho-Queiroz C, Wilding G, LoVerde PT. Nucleic acid vaccination with Schistosoma mansoni antioxidant enzyme cytosolic superoxide dismutase and the structural protein filamin confers protection against the adult worm stage. Infect Immun (2004) 72(10):6112-24. doi:10.1128/IAI.72.10.6112-6124.2004

32. Carvalho-Queiroz C, Cook R, Wang CC, Correa-Oliveira R, Bailey NA, Egilmez $\mathrm{NK}$, et al. Cross-reactivity of Schistosoma mansoni cytosolic superoxide dismutase, a protective vaccine candidate, with host superoxide dismutase and identification of parasite-specific B epitopes. Infect Immun (2004) 72(5):2635-47. doi:10.1128/ IAI.72.5.2635-2647.2004

33. Hartikka J, Sawdey M, Cornefert-Jensen F, Margalith M, Barnhart K, Nolasco $\mathrm{M}$, et al. An improved plasmid DNA expression vector for direct injection into skeletal muscle. Hum Gene Ther (1996) 7(10):1205-17. doi:10.1089/ hum.1996.7.10-1205

34. Soisson LA, Reid GD, Farah IO, Nyindo M, Strand M. Protective immunity in baboons vaccinated with a recombinant antigen or radiation-attenuated cercariae of Schistosoma mansoni is antibody-dependent. J Immunol (1993) 151(9):4782-9.

35. Kariuki TM, Van Dam GJ, Deelder AM, Farah IO, Yole DS, Wilson RA, et al Previous or ongoing schistosome infections do not compromise the efficacy of the attenuated cercaria vaccine. Infect Immun (2006) 74(7):3979-86. doi:10.1128/ IAI.01657-05

36. Katz N, Chaves A, Pellegrino J. A simple device for quantitative stool thick-smear technique in schistosomiasis mansoni. Rev Inst Med Trop Sao Paulo (1972) 14(6):397-400.

37. Farah IO, Nyindo M. Schistosoma mansoni induces in the Kenyan baboon a novel intestinal pathology that is manifestly modulated by an irradiated cercarial vaccine. J Parasitol (1996) 82(4):601-7. doi:10.2307/3283784

38. Nyindo M, Farah IO. The baboon as a non-human primate model of human schistosome infection. Parasitol Today (1999) 15(12):478-82. doi:10.1016/ S0169-4758(99)01569-0

39. Damian RT, de la Rosa MA, Murfin DJ, Rawlings CA, Weina PJ, Xue YP. Further development of the baboon as a model for acute schistosomiasis. Mem Inst Oswaldo Cruz (1992) 87(Suppl 4):261-9. doi:10.1590/S0074-02761992000800041

40. Siddiqui AA, Ahmad G, Damian RT, Kennedy RC. Experimental vaccines in animal models for schistosomiasis. Parasitol Res (2008) 102(5):825-33. doi:10.1007/ s00436-008-0887-6

41. Coulson PS, Kariuki TM. Schistosome vaccine testing: lessons from the baboon model. Mem Inst Oswaldo Cruz (2006) 101(Suppl 1):369-72. doi:10.1590/ S0074-02762006000900061

42. Kariuki TM, Farah IO, Yole DS, Mwenda JM, Van Dam GJ, Deelder AM, et al. Parameters of the attenuated schistosome vaccine evaluated in the olive baboon. Infect Immun (2004) 72(9):5526-9. doi:10.1128/IAI.72.9.5526-5529.2004 
43. O'Hagan DT, MacKichan ML, Singh M. Recent developments in adjuvants for vaccines against infectious diseases. Biomol Eng (2001) 18(3):69-85. doi:10.1016/ S1389-0344(01)00101-0

44. O'Hagan DT, Rahman D, McGee JP, Jeffery H, Davies MC, Williams P, et al. Biodegradable microparticles as controlled release antigen delivery systems. Immunology (1991) 73(2):239-42.

45. Okada H, Toguchi H. Biodegradable microspheres in drug delivery. Crit Rev Ther Drug Carrier Syst (1995) 12(1):1-99. doi:10.1615/CritRevTherDrugCarrierSyst. v12.i1.10

46. Mathiowitz E, Jacob JS, Jong YS, Carino GP, Chickering DE, Chaturvedi P, et al. Biologically erodable microspheres as potential oral drug delivery systems. Nature (1997) 386(6623):410-4. doi:10.1038/386410a0

47. Egilmez NK, Jong YS, Iwanuma Y, Jacob JS, Santos CA, Chen FA, et al. Cytokine immunotherapy of cancer with controlled release biodegradable microspheres in a human tumor xenograft/SCID mouse model. Cancer Immunol Immunother (1998) 46(1):21-4. doi:10.1007/s002620050455

48. Egilmez NK, Jong YS, Sabel MS, Jacob JS, Mathiowitz E, Bankert RB. In situ tumor vaccination with interleukin-12-encapsulated biodegradable microspheres: induction of tumor regression and potent antitumor immunity. Cancer Res (2000) 60(14):3832-7.

49. Rosas JE, Pedraz JL, Hernandez RM, Gascon AR, Igartua M, Guzman F, et al. Remarkably high antibody levels and protection against $P$. falciparum malaria in Aotus monkeys after a single immunisation of SPf66 encapsulated in PLGA microspheres. Vaccine (2002) 20(13-14):1707-10. doi:10.1016/ S0264-410X(01)00508-4

50. Shi L, Caulfield MJ, Chern RT, Wilson RA, Sanyal G, Volkin DB. Pharmaceutical and immunological evaluation of a single-shot hepatitis B vaccine formulated with PLGA microspheres. J Pharm Sci (2002) 91(4):1019-35. doi:10.1002/ jps. 10042

51. O'Hagan DT. Microparticles and polymers for the mucosal delivery of vaccines. Adv Drug Deliv Rev (1998) 34(2-3):305-20. doi:10.1016/S0169-409X(98)00045-3

52. Sun H, Pollock KG, Brewer JM. Analysis of the role of vaccine adjuvants in modulating dendritic cell activation and antigen presentation in vitro. Vaccine (2003) 21(9-10):849-55. doi:10.1016/S0264-410X(02)00531-5

53. Dupre L, Poulain-Godefroy O, Ban E, Ivanoff N, Mekranfar M, Schacht AM, et al. Intradermal immunization of rats with plasmid DNA encoding Schistosoma mansoni $28 \mathrm{kDa}$ glutathione S-transferase. Parasite Immunol (1997) 19(11):505-13. doi:10.1046/j.1365-3024.1997.d01-163.x

54. LoVerde PT, Carvalho-Queiroz C, Cook R. Vaccination with antioxidant enzymes confers protective immunity against challenge infection with Schistosoma mansoni. Mem Inst Oswaldo Cruz (2004) 99(5 Suppl 1):37-43. doi:10.1590/ S0074-02762004000900007

55. Butterworth AE. Immunological aspects of human schistosomiasis. Br Med Bull (1998) 54(2):357-68. doi:10.1093/oxfordjournals.bmb.a011693

56. Pearce EJ, MacDonald AS. The immunobiology of schistosomiasis. Nat Rev Immunol (2002) 2(7):499-511. doi:10.1038/nri843

57. Colley DG, Secor WE. Immunology of human schistosomiasis. Parasite Immunol (2014) 36(8):347-57. doi:10.1111/pim.12087

58. Correa-Oliveira R, Caldas IR, Gazzinelli G. Natural versus drug-induced resistance in Schistosoma mansoni infection. Parasitol Today (2000) 16(9):397-9. doi:10.1016/ S0169-4758(00)01740-3

59. Wynn TA, Hoffmann KF. Defining a schistosomiasis vaccination strategy - is it really Th1 versus Th2? Parasitol Today (2000) 16(11):497-501. doi:10.1016/ S0169-4758(00)01788-9

60. Allen JE, Maizels RM. Diversity and dialogue in immunity to helminths. Nat Rev Immunol (2011) 11(6):375-88. doi:10.1038/nri2992

61. Karmakar S, Zhang W, Ahmad G, Torben W, Alam MU, Le L, et al. Use of an Sm-p80-based therapeutic vaccine to kill established adult schistosome parasites in chronically infected baboons. J Infect Dis (2014) 209(12):1929-40. doi:10.1093/ infdis/jiu031
62. Wilson MS, Cheever AW, White SD, Thompson RW, Wynn TA. IL-10 blocks the development of resistance to re-infection with Schistosoma mansoni. PLoS Pathog (2011) 7(8):e1002171. doi:10.1371/journal.ppat.1002171

63. Bourke CD, Nausch N, Rujeni N, Appleby LJ, Mitchell KM, Midzi N, et al. Integrated analysis of innate, Th1, Th2, Th17, and regulatory cytokines identifies changes in immune polarisation following treatment of human schistosomiasis. J Infect Dis (2013) 208(1):159-69. doi:10.1093/infdis/jis524

64. Mola PW, Farah IO, Kariuki TM, Nyindo M, Blanton RE, King CL. Cytokine control of the granulomatous response in Schistosoma mansoni-infected baboons: role of exposure and treatment. Infect Immun (1999) 67(12):6565-71.

65. Labeaud AD, Malhotra I, King MJ, King CL, King CH. Do antenatal parasite infections devalue childhood vaccination? PLoS Negl Trop Dis (2009) 3(5):e442. doi:10.1371/journal.pntd.0000442

66. Malhotra I, McKibben M, Mungai P, McKibben E, Wang X, Sutherland LJ, et al. Effect of antenatal parasitic infections on anti-vaccine IgG levels in children: a prospective birth cohort study in Kenya. PLoS Negl Trop Dis (2015) 9(1):e0003466. doi:10.1371/journal.pntd.0003466

67. Butterworth AE, Dunne DW, Fulford AJ, Thorne KJ, Gachuhi K, Ouma JH, et al. Human immunity to Schistosoma mansoni: observations on mechanisms, and implications for control. Immunol Invest (1992) 21(5):391-407. doi:10.3109/08820139209069381

68. Chuah C, Jones MK, Burke ML, McManus DP, Gobert GN. Cellular and chemokine-mediated regulation in schistosome-induced hepatic pathology. Trends Parasitol (2014) 30(3):141-50. doi:10.1016/j.pt.2013.12.009

69. Souza PR, Souza AL, Negrao-Correa D, Teixeira AL, Teixeira MM. The role of chemokines in controlling granulomatous inflammation in Schistosoma mansoni infection. Acta Trop (2008) 108(2-3):135-8. doi:10.1016/j.actatropica.2008.04.016

70. Otoni A, Teixeira AL, Voieta I, Antunes CM, Costa Melo VL, Drummond SC, et al. Chemokine profile in the sera and urine of patients with schistosomal glomerulopathy. Am J Trop Med Hyg (2014) 90(1):48-53. doi:10.4269/ajtmh.13-0270

71. Ahmed H, Romeih M, Abou Shousha T, Dabaa EEM, Saber MA. DNA immunization with the gene encoding $\mathrm{Sm} 21.7$ protein protects mice against Schistosoma mansoni infections. J Am Sci (2006) 2:59-69.

72. Ahmad G, Zhang W, Torben W, Damian RT, Wolf RF, White GL, et al. Protective and antifecundity effects of Sm-p80-based DNA vaccine formulation against Schistosoma mansoni in a nonhuman primate model. Vaccine (2009) 27(21):2830-7. doi:10.1016/j.vaccine.2009.02.096

73. Capron A, Capron M, Dombrowicz D, Riveau G. Vaccine strategies against schistosomiasis: from concepts to clinical trials. Int Arch Allergy Immunol (2001) 124(1-3):9-15. doi:10.1159/000053656

74. Boulanger D, Reid GD, Sturrock RF, Wolowczuk I, Balloul JM, Grezel D, et al. Immunization of mice and baboons with the recombinant Sm28GST affects both worm viability and fecundity after experimental infection with Schistosoma mansoni. Parasite Immunol (1991) 13(5):473-90. doi:10.1111/j.1365-3024.1991. tb00545.x

75. Siddiqui AA, Pinkston JR, Quinlin ML, Saeed Q, White GL, Shearer MH, et al. Characterization of the immune response to DNA vaccination strategies for schistosomiasis candidate antigen, Sm-p80 in the baboon. Vaccine (2005) 23(12):1451-6. doi:10.1016/j.vaccine.2004.09.018

Conflict of Interest Statement: The authors declare that the research was conducted in the absence of any commercial or financial relationships that could be construed as a potential conflict of interest.

Copyright (C) 2015 Carvalho-Queiroz, Nyakundi, Ogongo, Rikoi, Egilmez, Farah, Kariuki and LoVerde. This is an open-access article distributed under the terms of the Creative Commons Attribution License (CC BY). The use, distribution or reproduction in other forums is permitted, provided the original author(s) or licensor are credited and that the original publication in this journal is cited, in accordance with accepted academic practice. No use, distribution or reproduction is permitted which does not comply with these terms. 